\title{
$\mathrm{M}|\mathrm{R}| \mathrm{S}$ Internet Journal Nitride Semiconductor Research
}

\section{Review of Structure of Bare and Adsorbate-Covered GaN(0001) Surfaces}

\author{
R. M. Feenstra ${ }^{1}$, J. E. Northrup ${ }^{2}$ and Jörg Neugebauer ${ }^{3}$ \\ ${ }^{1}$ Department of Physics, Carnegie Mellon University, \\ ${ }^{2}$ Palo Alto Research Center, \\ ${ }^{3}$ Fritz-Haber-Institut der MPG,
}

(Received Friday, March 22, 2002; accepted Wednesday, May 1, 2002)

\begin{abstract}
A review of surface structures of bare and adsorbate-covered GaN (0001) and (000) surfaces is presented, including results for $\mathrm{In}, \mathrm{Mg}, \mathrm{Si}$, and $\mathrm{H}$ adsorbates. Emphasis is given to direct determination of surface structure employing experimental techniques such as scanning tunneling microscopy, electron diffraction, and Auger electron spectroscopy, and utilizing first principles computations of the total energy of various structural models. Different surface stoichiometries are studied experimentally by varying the surface preparation conditions (e.g. Ga-rich compared to Nrich), and the stoichiometry is included in the theory by performing calculations for various chemical potentials of the constituent atoms. Based on the work reviewed here, surface reconstructions for plasma-assisted molecular beam epitaxy growth of GaN (0001) and (0001) surfaces are fairly well understood, but reconstructions for reactive molecular beam epitaxy or for metal-organic vapor phase epitaxy (both involving $\mathrm{H}$, at moderate and high temperatures, respectively) are less well understood at present.
\end{abstract}

\section{Introduction}

Gallium nitride and other III-nitrides have attracted considerable interest recently because of their applications for blue light-emitting diodes and lasers and for high frequency/high power transistors [1] [2] [3] [4] [5] [6] [7]. These materials have several unique properties compared to the more conventional III-V semiconductors (GaAs, InP, etc.): they exist in both cubic (zincblende) and hexagonal (wurtzite) form, they are refractory, and some of the materials have large band gaps. The relatively small size of nitrogen, compared to Ga or In, in these compounds leads to a number of unique surface structures, which have been explored in several papers for the (001) growth surface of cubic GaN [8] [9] [10]. For the technologically more relevant (0001) growth surface of hexagonal GaN, reports over the past 5 years have led to considerable understanding of its structure, for both bare and adsorbate covered surfaces. This article presents a review of these studies of the surface science of wurtzite GaN (0001) and (0001) surfaces. It is important to understand the surface structures of these materials, since this knowledge will impact our ability to achieve high quality epitaxial growth of the materials as required for optoelectronic and electronic applications.

Common growth methods for GaN films include metal-organic vapor phase epitaxy (MOVPE) and molecular beam epitaxy (MBE). The latter can be performed using a plasma source for nitrogen (plasmaassisted MBE, or PAMBE) or using ammonia, which thermally decomposes on the growth surface (reactive MBE, or RMBE). The MBE technique by virtue of its ultra-high vacuum apparatus is more amenable to surface science studies, and most surface science work to date has been performed on samples grown by PAMBE. Such work constitutes the bulk of the material reviewed in this article. Reports of the surface science of $\mathrm{GaN}$ films grown by RMBE have also appeared [11] [12] [13], and one group has reported surface science results for MOVPE-grown material [14]. Those works are discussed below in Section 3.5.

It is important to note that the $(0001)$ and $(000 \overline{1})$ directions of $\mathrm{GaN}$ are inequivalent, as illustrated in Figure 1 (by convention, the positive (0001) direction is given by a vector pointing from a $\mathrm{Ga}$ atom to a nearestneighbor $\mathrm{N}$-atom along (0001)). Thin films having either polarity have been grown. Films with (0001) sur- 
face normal are called Ga-polar, and those with $(000 \overline{1})$ surface normal are called N-polar. The surfaces of such films are sometimes called Ga-face or $\mathrm{N}$-face, respectively. The identification of film polarity, and the factors influencing the nucleation of a particular polarity, are discussed in the early review by Hellman et al. [15]. Two additional issues which have been addressed since that article are: (a) for MBE growth on sapphire, the formation of Ga- or N-polarity is largely influenced by the choice of AlN or GaN buffer layers used for the initial film nucleation [11] [16] [17] and (b) the observation of a clear $2 \times 2$ reconstruction during PAMBE growth of Ga-polar material (which is argued by Hellman to provide a means of identifying the Ga-polarity) has been recently argued to arise from the unintentional presence of arsenic on the growth surface [18] [19], as further discussed below. This arsenic occurs in a number of growth systems since they were previously used for MBE growth of GaAs, and the influence of residual arsenic has also been established for the case of cubic GaN [8] [9] [10]. However, notwithstanding the role of arsenic, it should be noted that even comparing results from arsenic-free growth systems leads in some cases to disagreement in the precise reconstructions reported; it is clear that for certain conditions (e.g. intermediate stoichiometries) the GaN surface symmetry can be modified by rather low levels of contamination.

Regarding nomenclature, we repeat here the recommendation made by Hellman et al., that the terms "Gaterminated" or "N-terminated" should be avoided when referring to film or surface polarity. The actual termination of a film depends, of course, on the surface reconstruction and surface stoichiometry. These terms, Ga- or $\mathrm{N}$-terminated, will however be occasionally used below when referring to the actual presence of a particular terminating layer of atoms on a surface.

In this article we review results for surface reconstructions of $\mathrm{GaN}(000 \overline{1})$ and (0001) surfaces, focusing first on reconstructions of the bare surfaces and then discussing the addition of various adlayers including In, $\mathrm{Mg}, \mathrm{Si}$, and $\mathrm{H}$ to the surfaces. The kinetics of the surface atoms during growth is also discussed.

\section{Bare Surfaces}

\subsection{N-polarity}

\subsubsection{Experiment}

A number of early works have reported surface symmetries other than $1 \times 1$ for $\mathrm{GaN}$ surfaces, but the nature of these reconstructions was completely unknown [15] [20] [21] [22] [23] [24] [25] [26] [27] [28] [29]. The first paper to determine geometrical arrangements of reconstructions was the work of Smith et al. for the $\mathrm{GaN}(000 \overline{1})$ surface [30]. These films were grown by
PAMBE on sapphire. Their polarity was not known prior to the study; an outcome of the work was the determination of N-polarity, and subsequent studies by other workers upheld this conclusion [11]. The surface structure varies with surface stoichiometry. Figure 2 shows scanning tunneling microscopy (STM) images of the four most common reconstructions: $1 \times 1,3 \times 3,6 \times 6$, and $c(6 \times 12)$, in order of increasing $\mathrm{Ga}$ coverage.

The $1 \times 1$ reconstruction appears as a hexagonal array of corrugation maxima, with a lateral spacing equal to the c-plane lattice constant of GaN, $3.19 \AA$. The $3 \times 3$ is similar in appearance but displays an asymmetry within the unit cell as well as additional structure at lower biases. The asymmetry of the unit cell reflects the fact that each GaN bilayer has only three-fold symmetry. STM images confirm that this asymmetry reverses upon descending a single bilayer-high step on the surface. The $6 \times 6$ is made up of ring-shaped structures. Each ring has three-fold symmetry with lobes from three neighboring rings coming close together. This results in two different kinds of "holes" around the rings, one appearing deeper than the other. The $c(6 \times 12)$ reconstruction is qualitatively different in appearance from the previous three. Row-like structures are observed running parallel to $\langle 1 \overline{1} 00\rangle$ directions of the crystal. Circular corrugation maxima appear in pairs along the rows; there are two possible angular orientations of these pairs of maxima with respect to the row directions in addition to the three possible row directions. Voltage dependence of the STM images for each reconstruction has been studied; no strong dependence is observed, except for the $c(6 \times 12)$ structure where the appearance of the row-like features differs between empty and filled states [31].

For determining structural models of the observed reconstructions, an important constraint is the number of $\mathrm{Ga}$ (and N) atoms involved in each structure. The observed surface reconstructions form when the surface is Ga-rich [11] [30]. Formation of the $3 \times 3$ reconstruction was found to require $0.145 \pm 0.025 \mathrm{ML}$ (ML = monolayer $=1.14 \times 10^{15}$ atoms $/ \mathrm{cm}^{2}$ ) more Ga than the $1 \times 1$ structure, corresponding to $1.3 \pm 0.2$ atoms per $3 \times 3$ unit cell. The $6 \times 6$ and $c(6 \times 12)$ require additional amounts of $\mathrm{Ga}$, estimated to be 0.43 and $0.58 \mathrm{ML}$ respectively relative to the $1 \times 1$ surface [31]. It is important to note that this "excess" Ga (over that required for the $1 \times 1$ ) is only weakly bound onto the surface. Above a temperature of about $200^{\circ} \mathrm{C}$, the $3 \times 3,6 \times 6$, and $\mathrm{c}(6 \times 12)$ all transform reversibly to a $1 \times 1$ arrangement believed to consist simply of the $1 \times 1$ Ga-terminated surface (described below) together with the excess $\mathrm{Ga}$ in a mobile, disordered (probably lattice gas) arrangement on the surface. 


\subsubsection{Theory}

First principles total energy calculations of the relative stability of possible models of surface reconstructions can provide definitive determinations of the surface structure provided certain conditions are met. One requirement is that the system under consideration must be reasonably close to equilibrium. It is not obvious $a$ priori that this requirement is satisfied in general by surface structures prepared by an MBE growth process. Nevertheless theorists have moved forward using a thermodynamic approach and there is now a widespread consensus that it is possible to obtain a reasonably complete mapping of the observed reconstructions by calculating the surface formation energies as a function of one or more of the atomic chemical potentials of the constituents. Prototypical examples of systems where this approach has achieved some success include the $\mathrm{GaAs}(001)$ and $\mathrm{ZnSe}(001)$ surfaces. The approach has also been successful in chemisorption systems such as $\mathrm{Si}(001) \mathrm{H}$. One of the objectives of work on GaN surfaces is to determine if the same theoretical approach will be successful for GaN and the other highly ionic wide-band-gap materials. At present the theoretical mapping for $\mathrm{GaN}$ is not complete: some of the observed reconstructions - such as the $6 \times 6$ and $c(6 \times 12)$ on the $(000 \overline{1})$ surface and the $5 \times 5$ and $6 \times 4$ on the $(0001)$ surface discussed below - are too large and complex for theoretical analysis at this time. A second requirement is that a sufficiently large number of structural models should be considered. Input from experiment is crucial, both in suggesting possible structures and limiting the number of models that must be considered.

For $\mathrm{GaN}$ one calculates the relative formation energies of surfaces as a function of the $\mathrm{Ga}$ and $\mathrm{N}$ chemical potentials $\left(\mu_{\mathrm{Ga}}\right.$ and $\left.\mu_{\mathrm{N}}\right)$. The formation energy of a system comprised of $\mathrm{Ga}$ and $\mathrm{N}$ is defined as $\Omega=\mathrm{E}-$ $\mathrm{n}_{\mathrm{Ga}} \mu_{\mathrm{Ga}}-\mathrm{n}_{\mathrm{N}} \mu_{\mathrm{N}}$. In this expression $\mathrm{E}$ is the total energy per supercell that is calculated for a structure containing the specified number of gallium and nitrogen atoms $\left(\mathrm{n}_{\mathrm{Ga}}, \mathrm{n}_{\mathrm{N}}\right)$. We assume equilibrium with bulk GaN: This implies the relation $\mu_{\mathrm{Ga}}+\mu_{\mathrm{N}}=\mu_{\mathrm{GaN}(\text { bulk) }}$, where $\mu_{\mathrm{GaN} \text { (bulk) }}$ is the calculated energy per Ga-N pair for wurtzite GaN. This relation between the chemical potentials is used to eliminate either the gallium or nitrogen chemical potential as an independent variable and to write the formation energy as a function of a single chemical potential. The formation energy may then be written as $\Omega=\mathrm{E}_{\mathrm{ga}}-\left(\mathrm{n}_{\mathrm{Ga}}-\mathrm{n}_{\mathrm{N}}\right)\left(\mu_{\mathrm{Ga}}-\mu_{\mathrm{Ga}(\text { bulk })}\right)$, where $\mathrm{E}_{\mathrm{ga}}$ is the formation energy corresponding to the Ga-rich limit, where by definition $\mu_{\mathrm{Ga}}=\mu_{\mathrm{Ga} \text { (bulk). }}$. The relative energy difference between any two reconstructions may then be expressed as $\Delta \Omega=\Delta \mathrm{E}_{\mathrm{ga}}-\left(\Delta \mathrm{n}_{\mathrm{Ga}}-\right.$
$\left.\Delta \mathrm{n}_{\mathrm{N}}\right)\left(\mu_{\mathrm{Ga}}-\mu_{\mathrm{Ga}(\mathrm{bulk})}\right)$. The $\mathrm{Ga}$ chemical potential is bounded from above by $\mu_{\mathrm{Ga}(\text { bulk) }}$ and from below by $\mu_{\mathrm{Ga}(\text { bulk })}-|\Delta \mathrm{H}|$ where $\Delta \mathrm{H}$ is the heat of formation of $\mathrm{GaN}$. We refer to the two endpoints of the allowed $\mathrm{Ga}$ chemical potential, $\mu_{\mathrm{Ga}}=\mu_{\mathrm{Ga} \text { (bulk) }}$ and $\mu_{\mathrm{Ga}}=\mu_{\mathrm{Ga}(\text { bulk) }}-$ $|\Delta \mathrm{H}|$, as the Ga-rich and N-rich limits. The thermodynamically allowed structures are those that have the lowest energy for some value of the Ga chemical potential within the allowed range [32].

Total energy calculations for the $(000 \overline{1})$ surface shown in Figure 3(b) [30], indicate that the only feasible candidate for the experimentally observed $1 \times 1$ structure is the Ga adlayer model. This structure is shown in Figure 4(a) [30]. In this structure each $\mathrm{Ga}$ atom is positioned in an atop site with vertical N-Ga bonds of length $1.99 \AA$. The atop registry of the Ga atoms is preferred by a wide margin over registrations in which the $\mathrm{Ga}$ is located over T4 or H3 sites. Electronic structure calculations predict that the Ga adlayer structure gives rise to highly dispersive bands of surface states inside the bulk band gap [33]. A recent experimental study by Ryan et al. [34] appears to corroborate the existence of such highly dispersive states. The correspondence between the theory and the photoemission experiment is not complete, however, and further work is required to account for all the features seen in the experiment.

In N-rich conditions the most stable structure that has been obtained theoretically is the $2 \times 2 \mathrm{Ga}$ adatom model, with the adatom in an $\mathrm{H} 3$ site. The adatom forms three bonds to the $\mathrm{N}$ atoms in the layer below, and the length of these bonds is $1.98 \AA$. So far, a $2 \times 2$ structure has not been observed on the $\mathrm{GaN}(000 \overline{1})$ surface. The ideal $1 \times 1$ surface having one threefold coordinated $\mathrm{N}$ atom in each unit cell is not stable for any allowed value of the $\mathrm{Ga}$ chemical potential.

The $\mathrm{Ga}$ atoms in the $1 \times 1$ adlayer structure are each bonded to a single $\mathrm{N}$ atom and are separated from 6 other $\mathrm{Ga}$ atoms in the adlayer by a distance corresponding to a second-nearest-neighbor distance of bulk GaN, i.e. they are separated by about $3.2 \AA$. The relative stability of this type of structure is, at first sight, very surprising. To establish the plausibility of this structure a set of calculations was performed in order to determine how much of the binding energy of a $\mathrm{Ga}$ atom arises from intra-adlayer $\mathrm{Ga}-\mathrm{Ga}$ interactions and how much arises from the Ga-N bonds [33]. Starting from a $\mathrm{GaN}(000 \overline{1}) 1 \times 1 \mathrm{~N}$-terminated surface and a collection of isolated $\mathrm{Ga}$ atoms, the energy of the system decreases by $4.0 \mathrm{eV}$ per $\mathrm{Ga}$ atom when the adlayer is formed. Of this $4.0 \mathrm{eV} /$ atom, a $2.2 \mathrm{eV}$ reduction can be attributed to the formation of the Ga-N bonds and $1.8 \mathrm{eV}$ can be attributed to the formation of the Ga-Ga bonds within the adlayer. The first point to be made is that the $4.0 \mathrm{eV} /$ 
atom energy reduction is larger than the cohesive energy of bulk $\mathrm{Ga}(2.8 \mathrm{eV} /$ atom $)$. In other words the $1 \times 1 \mathrm{Ga}$ adlayer is much more stable than a surface with $\mathrm{Ga}$ droplets residing on a $\mathrm{N}$-terminated $1 \times 1$ surface. The second point is that a substantial fraction - about $45 \%$ of the binding energy of the $\mathrm{Ga}$ atoms can be attributed to intra-adlayer Ga-Ga bonding. For perspective, consider the analogous (hypothetical) reaction for $\mathrm{Ga}$ on an As-terminated $\mathrm{GaAs}(\overline{111}) 1 \times 1$ surface. The $\mathrm{Ga}$ atoms in such an adlayer would be separated from each other by about $4.0 \AA$ A. Now, the calculated energy reduction of the system that results from bringing isolated $\mathrm{Ga}$ atoms onto the As-terminated surface is found to be $2.4 \mathrm{eV} /$ atom. Of this $2.4 \mathrm{eV}$, a $1.6 \mathrm{eV}$ reduction arises from the $\mathrm{Ga}-\mathrm{As}$ bond and $0.8 \mathrm{eV}$ arises from the intra-adlayer $\mathrm{Ga}-\mathrm{Ga}$ bonds. In this case, since the cohesive energy of $\mathrm{Ga}$ is $2.8 \mathrm{eV} /$ atom, a $1 \times 1 \mathrm{Ga}$ adlayer would actually be unstable with respect to formation of $\mathrm{Ga}$ droplets on a $1 \times 1$ As-terminated surface. It is important to note that the energy reduction arising from intra-adlayer $\mathrm{Ga}-\mathrm{Ga}$ bonding is only $0.8 \mathrm{eV} /$ atom - just about $33 \%$ of the total reduction. It was found that the strength of the intra-adlayer Ga-Ga bonding increases by about $1.0 \mathrm{eV} /$ atom as the Ga-Ga separation in the adlayer is reduced from $4.0 \AA$ (for GaAs) to $3.2 \AA$ (for $\mathrm{GaN}$ ). The increased strength of the intra-adlayer Ga-Ga bonding is an important part of the reason why Ga adlayer structures can occur on $\mathrm{GaN}(000 \overline{1})$ surfaces, but not on the $\operatorname{GaAs}(\overline{111})$ surface.

The other observed reconstruction of the $\mathrm{GaN}(000 \overline{1})$ surface which has been examined is the $3 \times 3$ structure [30]. The preferred model is obtained by adding a single $\mathrm{Ga}$ atom per $3 \times 3$ unit cell to the $1 \times 1 \mathrm{Ga}$ adlayer model. This additional $\mathrm{Ga}$ adatom is located in a hollow site $0.75 \AA$ above its three neighboring Ga atoms as shown in Figure 4(b). To accommodate the additional atom the three neighboring $\mathrm{Ga}$ atoms relax laterally away from the adatom by more than $0.5 \AA$ so that the length of the three Ga-Ga bonds is $2.50 \AA$. This structure is indeed found to be energetically favorable compared to the $1 \times 1$ adlayer model in Ga-rich conditions (those results are not shown in Figure 3 since they were performed by including the $\mathrm{Ga} 3 \mathrm{~d}$ electrons as part of the core using the nonlinear core correction [30]). On the basis of these calculations it is concluded that the $(000 \overline{1})$ surface consists of an atop-registered $\mathrm{Ga}$ adlayer, and that this adlayer is decorated by additional Ga adatoms.

The atop-registered $1 \mathrm{x} 1$ adlayer model is also energetically favorable in the case of In-terminated $\mathrm{GaN}(000 \overline{1})$ surfaces, as discussed in more detail below. The In-In separation in bulk In is approximately $3.3 \AA$ and so $\mathrm{In}$ is a much larger atom than Ga. (In bulk Ga the corresponding Ga-Ga separation is $2.7 \AA$.) [a] In fact, the optimal In-In separation is slightly larger than the in- plane lattice constant of a $1 \times 1$ adlayer on the $(000 \overline{1})$ surface $(\sim 3.2 \AA)$. In contrast to the case of Ga adlayer structures, it appears that In atoms are too large to enable the insertion of additional atoms into the In adlayer to form stable adatom-on-adlayer structures. One may conclude that it is not likely that additional In adatoms can decorate a $1 \mathrm{ML}$ In adlayer termination of the $\mathrm{GaN}(000 \overline{1})$ surface. This conclusion is supported by total energy calculations showing that a $2 \times 2$ structure containing $1 / 4$ ML of In adatoms on the In adlayer is very high in energy [35].

\subsection{Ga-polarity}

\subsubsection{Experiment}

Reconstructions of the $\mathrm{GaN}(0001)$ surface have been reported by Smith et al. [36]. Those Ga-polar films were grown by PAMBE, using homoepitaxy on MOVPE-grown Ga-polar GaN, or using Si-polar $\mathrm{SiC}(0001)$ substrates. Compared to the $(000 \overline{1})$ surface, results for the (0001) surface are less well understood. In order of increasing Ga coverage, Smith et al. find $2 \times 2,5 \times 5,6 \times 4$, and " $1 \times 1$ " (pseudo- $1 \times 1$ ) structures.

The origin of the $2 \times 2$ surface reconstruction, in particular, is quite controversial. Smith et al. observe this structure only under N-rich conditions (it is not seen during growth, but it does appear when the Ga-flux is interrupted). They thus suggest that it arises from a $2 \times 2$ arrangement of $\mathrm{N}$ adatoms. In contrast, a number of groups have reported an intense $2 \times 2$ diffraction pattern during growth, as seen by reflection high energy electron diffraction (RHEED) [15] [25] [26] [37]. This pattern has been attributed to a $2 \times 2$ arrangement of Ga adatoms [15] [37]. Following an exhaustive but unsuccessful search for this $2 \times 2$ structure seen during growth, utilizing different growth conditions and multiple Nsources, Smith et al. finally proposed that it might arise from some unintentional adsorbate in the growth systems, e.g. arsenic [38]. Subsequent installation of an arsenic source into their MBE system led to an immediate detection of the intense $2 \times 2$ pattern (seen during growth), thus confirming its origin as being due to arsenic, as further discussed below in Section 3.3. Indeed, in several cases, the growth systems which reveal intense $2 \times 2$ patterns during PAMBE growth are known to have been previously used for GaAs growth [39] [40]. It is important to note that the above comments regarding the identity of the commonly observed $2 \times 2$ pattern apply only to PAMBE growth; for RMBE, a $2 \times 2$ pattern with different characteristics than that seen during PAMBE has been reported [13].

The $2 \times 2$ reconstruction observed by Smith et al. is prepared by nitriding the surface at a temperature of about $600^{\circ} \mathrm{C}$. STM images reveal a disordered surface with small domains of $2 \times 2$, consistent with the fact that 
the 1/2-order lines seen in RHEED are not very sharp [36]. From total energy calculations for the Ga face, two different $2 \times 2$ structures are found to be energetically favorable within certain ranges of the $\mathrm{Ga}$ chemical potential as described below: a N-adatom (H3) $2 \times 2$ and a Ga-adatom (T4) $2 \times 2$ [30]. The fact the $2 \times 2$ seen by Smith et al. is formed by nitridation led them to suggest that it arises from $\mathrm{N}$ adatoms.

The $5 \times 5$ reconstruction is obtained by annealing the Ga-face at $750^{\circ} \mathrm{C}$, depositing $1 / 2 \mathrm{ML}$ of $\mathrm{Ga}$, then reannealing the surface to about $700^{\circ} \mathrm{C}$ [36]. The surface obtained by annealing at $750^{\circ} \mathrm{C}$ alone is found to be disordered, but the $\mathrm{Ga}$ deposition and re-annealing process stabilizes the surface via the $5 \times 5$ reconstruction. Compared to reconstructions found on the $\mathrm{N}$-face, the Gaface $5 \times 5$ is strongly bias-dependent, suggestive of a semiconducting surface. Shown in Figure 5 is a pair of STM images of the $5 \times 5$ reconstruction acquired at positive sample bias (empty states) in (a), and negative sample bias (filled states) in (b), from nearby surface locations. At positive sample bias, the unit cells of the $5 \times 5$ can be readily identified by the dark trenches traversing the image in all three of the $\langle 11 \overline{2} 0\rangle$ directions. One $5 \times 5$ unit cell is marked in the image. Typically, four topographic maxima are observed within each unit cell. However, the height and shape of these maxima vary from one unit cell to the next. This lack of translational equivalence is even more evident at negative sample bias, where the topographic maxima appear to be grouped together on the surface into pairs, or in some cases, triplets. The more common pair features have a specific rotational orientation, namely along one of the $\langle 11 \overline{2} 0\rangle$ directions, with the particular orientation varying randomly over the surface.

Detailed analysis of the voltage-dependent images of the $5 \times 5$ leads to the model shown in Figure 6 . The topographic maxima seen in the images are interpreted in terms of $\mathrm{N}$ and $\mathrm{Ga}$ adatoms, residing on $\mathrm{T} 4$ and $\mathrm{H} 3$ sites respectively, together with some residual dangling bonds in the unit cell. A complete theoretical description of this surface is lacking at present, although some results are available for the relative stability of $\mathrm{Ga}$ and $\mathrm{N}$ adatoms on the surface as discussed in Section 2.2.2 below.

The $6 \times 4$ is formed by depositing $1 / 2 \mathrm{ML}$ of Ga onto the $5 \times 5$ and then briefly heating the surface up to $700^{\circ} \mathrm{C}$. Ga deposition alone will not produce the $6 \times 4$, suggesting that the formation of the $6 \times 4$ must involve extensive rearrangement of surface atoms. Surfaces showing clear $6 \times 4$ RHEED patterns obtained in this manner, however, are also found to contain large domains of $5 \times 5$, as shown in Figure 7. Seen there are STM image of both the $5 \times 5$ and $6 \times 4$ regions, at both positive sample bias and negative sample bias. As with the $5 \times 5$, the row-like
$6 \times 4$ structure shows a strong bias-dependence, again suggesting a semiconducting surface. At positive sample bias, each row is clearly defined by a line of bright features spaced $4 \times \mathrm{a}(\mathrm{a}=3.19 \AA)$ apart along the [11 $\overline{2} 0]$ direction, except where a structural defect breaks the periodicity. At negative sample bias, these maxima do not appear, but the rows are still clearly defined by a line of dark features

As seen in Figure 7 the $6 \times 4$ appears topographically lower, on the average, than the $5 \times 5$. This is counter-intuitive since the $6 \times 4$ is formed by adding Ga to the $5 \times 5$. One possible explanation is that the height difference is electronic in nature. However, this seems insufficient to explain the difference since the $5 \times 5$ is higher than the $6 \times 4$ at both positive sample bias (by $0.3 \AA$ ) and negative sample bias (by $0.4 \AA$ ). A second possibility is based on the observation that $6 \times 4$ surfaces not only contain $5 \times 5$ but also " $1 \times 1$ " [36]. This latter structure as described below is known to contain much more Ga compared to the other reconstructions, as measured by Auger electron spectroscopy (AES). The fact that all three reconstructions are found together suggests that the $5 \times 5$ and $6 \times 4$ may not be very different from each other in terms of energy, and possibly also Ga coverage. The " $1 \times 1$ ", on the other hand, appears to be energetically much more favorable, effectively acting as a $\mathrm{Ga}$ "sink". In any case, it is hard to imagine that the $6 \times 4$ could contain less Ga than the $5 \times 5$. The additional Ga in the $6 \times 4$ could form a structural arrangement allowing a denser packing of $\mathrm{Ga}$ compared to the $5 \times 5$. The observed height difference might then be explained by a combination of both structural and electronic effects.

As reported by Smith et al. [33] the most stable structure at high Ga coverage is one which displays a diffraction pattern dominated by $1 \times 1$ spots, so that this structure is known as pseudo- $1 \times 1$ or " $1 \times 1$ ". The structure can be formed in several ways, one of which is by depositing about $1 \mathrm{ML}$ of $\mathrm{Ga}$ onto the $6 \times 4$, followed by a rapid anneal to $700^{\circ} \mathrm{C}$. Another way to form the " $1 \times 1 "$ is to terminate the growth of GaN under slightly Ga-rich growth conditions. As the sample cools, the entire surface can become " $1 \times 1$ " although $5 \times 5$ and $6 \times 4$ may also be observed, depending on the precise amount of Ga present on the surface. The diffraction patterns of this surface show mainly $1 \times 1$ streaks (in RHEED) or spots (in low-energy electron diffraction, LEED), but includes sidebands or satellite spots in these patterns as described below. Hence this structure is referred to as " $1 \times 1$ ", using the quotation marks to indicate that the symmetry is not truly $1 \times 1$. During growth, this Ga-rich surface shows only $1 \times$ streaks, as illustrated in Figure $8(\mathrm{a})$. However, as the surface is cooled down to $<350^{\circ} \mathrm{C}$, distinct sidebands appear on the high wavevector sides of the firstorder streaks along the [11 $\overline{2} 0]$ azimuth, as shown in Fig- 
ure 8(b). Depending on the Ga coverage, the spacing of the sidebands from the first-order streaks at room temperature is either $0.16 \pm 0.01(\approx 1 / 6)$ or $0.08 \pm 0.01(\approx 1 /$ 12) of the $1 \times$ spacing $k_{1}=0.361 \AA^{-1}$, as illustrated by the two LEED patterns shown in Figs. 8(c,d). These structures are referred to as "1+1/6" and "1+ 1/12" respectively; the precise difference between these structures is not well understood at present. The $1+1 / 6$ structure, pictured in Figure 8(c), can exist down to room temperature for a narrow range of Ga coverage (just above that needed to form the $6 \times 4$ ), but for all higher coverages, the $1+1 / 6$ converts to $1+1 / 12$ as the temperature is reduced to about $200^{\circ} \mathrm{C}$.

The temperature dependence of the $1 \times 1$ surface is illustrated in Figs. 8(e-h), focusing on the vicinity of the integral order $(0,1)$ spot. Between room temperature and about $100^{\circ} \mathrm{C}$, as seen in Figure $8(\mathrm{e})$, a modulated ring of intensity with radius $0.08 k_{1}$ is observed around the $(0,1)$ spot with modulation at $60^{\circ}$ intervals. This ring has greater intensity on the high wavevector side of the spot. As the temperature is increased to about $150^{\circ} \mathrm{C}$, the ring modulation decreases slightly [Figure 8(f)]. As the surface temperature increases further to around $200^{\circ} \mathrm{C}$, the ring modulation decreases further [Figure $8(\mathrm{~g})$ ]. It is also seen that the radius of the ring appears to have decreased slightly to about $0.07 k_{1}$. As the temperature is raised past $200^{\circ} \mathrm{C}$, the pattern converts to $1+1 / 6$ (although not observed in this particular LEED experiment, the conversion from $1+1 / 12$ to $1+1 / 6$ in this temperature range has been observed consistently in RHEED experiments). Above $350^{\circ} \mathrm{C}$, one sees only the integral order LEED spot [Figure $8(\mathrm{~h})$ ]. This sequence of phase transitions is reversible. Thus we find that the ring modulation decreases with increasing temperature. At the same time, the ring radius decreases slightly from $0.08 k_{1}$ to $0.07 k_{1}$ with increasing temperature until about $200^{\circ} \mathrm{C}$, at which point it increases by a discrete amount to $0.16 k_{1}$.

The " $1 \times 1$ " diffraction patterns described above are typical of a incommensurate surface structure. The modulated ring structure and its temperature dependence indicate that this incommensurate structure possesses considerable dynamic, fluid-like character, even at room temperature. Thus, it was inferred that the " $1 \times 1$ " surface at room temperature is best characterized by a discommensuration-fluid phase [33], similar to that seen for $\mathrm{Au}(111)$ and $\operatorname{Pt}(111)$ at elevated temperatures [41]. Since the melting point of bulk Ga $\left(29.8^{\circ} \mathrm{C}\right)$ is very near room temperature, such a structural phase for this Garich surface is most reasonable. STM image for this surface generally do not display any atomic corrugation. Rather, the " $1 \times 1$ " regions appear quite featureless. Sometimes corrugation is seen, and in those cases it has precise $1 \times 1$ spacing (rather than some much longer corrugation, as would be expected from the diffraction results) [33]. This apparent discrepancy between the STM and diffraction results was resolved by postulating a model in which the surface contains two monolayers of excess $\mathrm{Ga}$ in an incommensurate arrangement, with this $\mathrm{Ga}$ existing in the mobile, fluid-like state at room temperature. Thus, the STM images reflect the time averaged position of the $\mathrm{Ga}$ atoms, which reflects the underlying $1 \times 1$ structure of the GaN bilayer below the excess Ga. In one exceptional case of STM imaging on the $(000 \overline{1})$ surface, a small reconstructed domain was observed in STM which has structure close to that expected for the " $1 \times 1$ ". It was speculated that this structure occurred at the top of an inversion domain and that the incommensurate arrangement of the " $1 \times 1$ " was frozen-in there due to the limited size of the domain [42].

STM images acquired at room temperature for a surface containing a mixture of " $1 \times 1$ ", $5 \times 5$, and $6 \times 4$ domains reveal a height difference of " $1 \times 1$ " islands relative to surrounding $6 \times 4$ and $5 \times 5$ regions of about $2.1 \AA$. Electronic effects can of course influence this height, but typically by only a few tenths of an $\AA$. As discussed above, the $5 \times 5$ and $6 \times 4$ regions are believed to contain adatoms with height (from theory [36]) of about $1.7 \AA$ above the $\mathrm{Ga}$ atoms in the outermost $\mathrm{GaN}$ bilayer. Thus, the thickness of the " $1 \times 1 "$ Ga layer is estimated to be about $3.8 \AA$, corresponding to about $1.8 \mathrm{ML}$. This estimate suggests that the " $1 \times 1 "$ reconstruction contains around 2 ML of excess Ga. Similarly, AES measurements indicate that the " $1 \times 1$ " surface contains $2-3$ additional ML of Ga above the outermost GaN bilayer [33]. Recent results of RHEED studies for Ga on $\mathrm{GaN}(0001)$ and $\operatorname{AlN}(0001)$ also yield a value for the stable coverage of $\mathrm{Ga}$ on those surfaces under Ga-rich conditions of about 2 ML [43].

The above diffraction results for the " $1 \times 1 "$ structure have recently also been clearly observed using low energy electron microscopy (LEEM) [44] [45]. Transitions between the $1+1 / 6$ and $1+1 / 12$ structures were found to occur reversibly, as a function of temperature and Ga coverage. The state of the surface was monitored in real-time during growth, and it was found, in agreement with prior work, that the presence of the Ga bilayer associated with the " $1 \times 1 "$ structure stabilizes the (0001) surface and gives rise to the flat morphology. Under Gapoor conditions the bilayer disappeared and the morphology became rough and microfaceted (for more discussion of this phenomena see Section 4). So long as the microfaceting was not too severe, it could be eliminated by restoring the $\mathrm{Ga}$ double layer during growth.

Based on the data of Smith et al. a model was proposed in which the " $1 \times 1$ " surface consists of a double layer of $\mathrm{Ga}$ atoms, with 7 unit cell of the Ga atoms resid- 
ing on 6 unit cells of the GaN [33]. The resulting spacing between the $\mathrm{Ga}$ atoms in the double layer is then close to what is ideally obtained in bulk $\mathrm{Ga}$, and in energy minimization calculations for free-standing $\mathrm{Ga}$ bilayers. This laterally contracted bilayer model is illustrated in Figure 9. Although a complete theoretical description of such a model is currently not possible (because a $6 \times 6$ unit cell would be required), a simplified version of the model with $\sqrt{3} \times \sqrt{3}$ symmetry has been shown to be energetically favorable under Ga-rich conditions [46], as further discussed in the following Section.

\subsubsection{Theory}

Let us now turn to theoretical results for structures on the (0001) surface. A large number of possible structures were considered theoretically by Smith et al. [30] and many could be ruled out on the grounds that they are thermodynamically unstable. A simple $1 \times 1 \mathrm{Ga}$ terminated N-Ga bilayer model (i.e. the "ideal" surface) can certainly be excluded. In this structure the $\mathrm{Ga}$ atoms at the surface are threefold coordinated - each $\mathrm{Ga}$ is bonded to three $\mathrm{N}$ atoms and there is one Ga dangling bond per atom. The total energy calculations shown in Figure 3(a) indicate that there is no region of the chemical potential space for which this structure is stable. Adding one monolayer of $\mathrm{Ga}$ to this $1 \times 1 \mathrm{Ga}$-terminated bilayer leads to the $1 \times 1 \mathrm{Ga}$ adlayer structures. Several possible adlayer registries (H3, T4, atop) were considered but in no case was a thermodynamically stable structure found. It is interesting to note that the surface energies of these $\mathrm{Ga}$ adlayer structures are rather insensitive to the registry. This has implications for the existence of stable incommensurate adlayer structures discussed below. After an extensive search it was ultimately surmised that there exists no stable structure having a true $1 \times 1$ symmetry for the clean $\mathrm{GaN}(0001)$ surface [30]. It was also determined that the $2 \times 2 \mathrm{Ga}$ vacancy model is not stable on the (0001) surface. This structure is slightly higher in energy than the N-H3 adatom model. The instability of the Ga vacancy model for $\mathrm{GaN}(0001)$ is interesting in view of the fact that a $2 \times 2$ Ga vacancy structure is known to exist on the GaAs(111) surface [47] [48].

On the basis of the results shown in Figure 3(a) only the $2 \times 2 \mathrm{~N}$ adatom and the $2 \times 2 \mathrm{Ga}$ adatom survive as potential candidates as thermodynamically stable structures on the $\mathrm{GaN}(0001)$ surface. The question then is which, if either, of these two models corresponds to the $2 \times 2$ structure that is observed. Given that the $2 \times 2$ structure seen in experiment is the least Ga-rich of all the observed reconstructions, and that it is often observed following an interruption in the Ga flux, it seems more likely that the observed $2 \times 2$ structure arises from $\mathrm{N}$ rather than $\mathrm{Ga}$ adatoms.
Of the two possible adsorption sites for the $\mathrm{N}$ adatom, the $\mathrm{H} 3$ site is preferred over the T4 site in the calculations by $0.7 \mathrm{eV} /(2 \times 2)$ cell [36]. A qualitatively similar result has been predicted for $\mathrm{N}$ adatom structures on the AlN(0001) surface, where the preference for the $\mathrm{H} 3$ site is $3.3 \mathrm{eV} /(2 \times 2)$ cell [49]. As yet there exist no experimental determinations of the site preference of $\mathrm{N}$ adatoms on either $\mathrm{GaN}$ or AlN surfaces. In the case of the $2 \times 2 \mathrm{Ga}$ adatom model a slight preference for the $\mathrm{T} 4$ site is predicted: the energy difference is about $0.12 \mathrm{eV} /$ $(2 \times 2)$ cell [36]. The different site preference exhibited by the $\mathrm{N}$ and $\mathrm{Ga}$ adatoms on the (0001) surface is related to their very different ionicities.

Concerning the Ga-adatom $2 \times 2$, it appears that it has a higher energy than the $5 \times 5$ and " $1 \times 1 "$ structures (described below), so that the Ga-adatom $2 \times 2$ is not actually energetically allowed (even though it is employed in many theoretical computations since it is a relatively simple structure).

Let us now consider the pseudo- $1 \times 1$ structure that is observed under very Ga-rich conditions. Although the STM corrugation pattern exhibits $1 \times 1$ symmetry the electron diffraction patterns obtained at temperatures less than $350^{\circ} \mathrm{C}$ reveal, in addition to the $1 \times 1$ spots, additional diffraction intensity in satellite spots [33]. These diffraction patterns indicate that the lattice vectors of the unit cell are in fact larger than those corresponding to a $1 \times 1$ cell, and furthermore it is found from Auger spectroscopy that the structure contains 2-3 ML of $\mathrm{Ga}$ in excess of that expected for a bulk terminated $\mathrm{GaN}$ surface. To explain these observations a laterally contracted Ga bilayer model has been proposed [46], shown in Figure 10. In this model the atoms in layer 1 are located in atop sites and are separated by $\mathrm{a}=3.19 \AA$. However, the atoms in the top layer (layer 0) are contracted. This lateral contraction of the top layer is proposed because calculations have shown that a lateral contraction of such an adlayer - so that the Ga-Ga separation is reduced from 3.19 to $2.75 \AA$ - is energetically favorable. This reduction in the spacing results in an increased density of atoms in the adlayer and therefore provides a natural explanation of the Auger data, which is suggestive of an increased Ga content (greater than 2 ML) on the surface. Total energy calculations for such an adlayer were performed using a $\sqrt{3} \times \sqrt{ } 3$ unit cell. Different registries of layer 0 were considered, as shown in Figure 10, but the energies were found to be independent, within $0.01 \mathrm{eV} / \mathrm{atom}$, of the registry. As seen in Figure 11 the laterally contracted bilayer structure becomes energetically favorable with respect to the $\mathrm{Ga}$ adatom model in Ga-rich conditions. This increase in stability has been traced to the energy benefit of reducing the in-plane $\mathrm{Ga}-\mathrm{Ga}$ spacing [33]. 


\section{Adsorbates}

\subsection{Indium}

InGaN is the dominant alloy material used for III-nitride light emitters. The larger size of indium compared to gallium leads to a variety of phenomena, including surface segregation, alloy phase separation, inhomogeneous incorporation, etc. Surface studies of InGaN have been performed by Chen et al. for both $(000 \overline{1})$ and (0001) surfaces [35] [50] [51] [52]. For the (0001) surface it was found that In substitutes for $\mathrm{Ga}$ in the surface layer. STM results are shown in Figure 12, for a sample prepared by growth at $670^{\circ} \mathrm{C}$ with $\mathrm{In} /(\mathrm{In}+\mathrm{Ga})$ flux ratio of $20 \%$. An X-ray diffraction (XRD) scan of this sample showed no InGaN peak, meaning that there is negligible indium incorporation in the bulk. However, AES indicated a significant amount of indium on the surface, with In/Ga Auger emission ratio of about 0.5. In the middle of the STM image, there is a step, and the image shows two separate terraces. Each terrace displays two types of regions, one bright and the other dark. In this type of STM constant-current image, roughly speaking, the bright areas are higher in morphology and dark areas are lower. As can be seen from the line cut, the height difference between bright and dark area is $0.30 \pm 0.05$ A.

Theoretical computations permit the identification of the bright and dark areas in the STM image. Those results are pictured in Figure 13. The total energy calculation is performed for various $\mathrm{GaN}(000 \overline{1}) 1 \times 1: \mathrm{Ga}_{\mathrm{y}} \mathrm{In}_{1-\mathrm{y}}$ adlayer structures. The calculations are performed in a $2 \times 2$ unit cell with various numbers of In and $\mathrm{Ga}$ atoms in the top site. The equilibrium adlayer In-N bond length is found to be about $2.23 \AA$ while the equilibrium Ga-N bond in the adlayer is $1.99 \AA$. These values are essentially independent of $y$. From explicit examination of charge density contours for states located near the Fermi-level, it is found that the different height of the adatoms above the surface $(\sim 0.2 \AA)$ is manifested in a corrugation in the charge density of a similar magnitude at a height of several $\AA$ above the adatoms. The bright regions in the STM image are thus identified as arising from indium atoms in the surface adlayer, and the dark regions as arising from gallium atoms in the adlayer.

STM results for the InGaN(0001) surface are shown in Figure 14. The films shown there were known from AES measurements to have surface indium coverage of about $1 \mathrm{ML}$, and bulk indium content of several \% or less. Figure 14(a), shows several regions of apparently different structure. In the lower right hand corner is a region of uniform, $1 \times 1$ corrugation. Elsewhere on the surface are seen areas of brighter (higher) corrugation, with $1 \times$ and $2 \times$ spacing, and in these regions of brighter corrugation the presence of small black vacancy islands is observed. Total energy calculations performed for a large number of $\mathrm{InGaN}(0001)$ surfaces indicate that a $1 \times 1$ surface with $1 \mathrm{ML}$ of In in atop sites (layer 1 in Figure 14) is energetically favorable under In and Ga-rich conditions [53]. This structure was associated with the $1 \times 1$ region seen in the lower right hand corner of Figure 14. For more Ga-deficient conditions the calculations show that it becomes energetically favorable to incorporate some In atoms into layer 2 as well. The height of the observed bright maxima, typically $0.2 \AA$ above the nominal height of the $1 \times 1$ region, is consistent with the calculated $0.3 \AA$ increase in height of a layer 1 atom produced by substitution of In for Ga in layer 2. The bright corrugation maxima observed elsewhere in the image were thus attributed to the presence of layer 2 In-atoms.

The surfaces pictured in Figs. 14(b) and (c) contain slightly higher indium than that of Figure 14(a), and in this case the surface phase containing vacancy islands covers the entire surface. The vacancy islands appear dark (lower surface height) for both positive and negative sample bias voltage, indicating that atoms are indeed missing from those areas. The vacancy islands do not grow with time, but rather, they have an equilibrium diameter of 10-20 $\AA$. The depth of the smallest vacancy islands seen in Figure 14 is typically $0.8 \AA$, but this value is probably limited by the shape of the STM probe tip. For the larger vacancy islands, a depth of about 2.5 $\AA$ was found, indicating that at least one layer of atoms is missing from the surface. As seen in Figure 14(c), bright rings of atoms are sometimes (depending on imaging condition) see surrounding the vacancy islands. These bright features were attributed to In atoms at the edge of the islands, as revealed in the theoretical analysis below.

In order to identify the underlying mechanism giving rise to the observed structural instabilities, first-principles total energy calculations for a variety of InGaN(0001) surface structures were performed [51]. Because InGaN films must be grown under very N-rich (Ga-deficient) conditions to obtain In concentrations greater than a few percent, attention was focussed on surface structures that could be stable under Ga-deficient conditions [53]. Specifically, surfaces were considered in which layer 1 is completely occupied by In atoms and layer 2 is occupied by both In and Ga. Structures of this type are indicated schematically in Figs. 14 and 15. Because the In-N bond is about $0.23 \AA$ longer than the Ga-N bond, partial occupation of the second layer by In leads to a substantial surface strain. It was found that for sufficiently large In concentrations in layer 2 it is energetically favorable to create $\mathrm{N}$ vacancies in layer 3. For example, for the structure shown in Figure 15 (a) containing 7/4 ML of In (1 ML in layer 1 and $3 / 4 \mathrm{ML}$ in layer 2), it was found that the creation of a $\mathrm{N}$ - 
vacancy in layer 3 of this structure, as shown in Figure 14(b), is exothermic. The energy of the reaction depends on the $\mathrm{N}$ chemical potential, but is at least $0.85 \mathrm{eV}$ per vacancy. The exothermicity of vacancy formation in a system having a full monolayer of In atoms in layer 2 is even greater, being at least $1.07 \mathrm{eV}$ per vacancy. It is important to note that in both of these exothermic reactions each of the three layer 2 sites adjacent to the vacancy is occupied by an In atom. The relative weakness of the In-N bonds compared to Ga-N is an important component in this structural instability.

Given the above results it is quite plausible that the vacancy islands seen in the STM images form in order to relieve surface strain and permit the segregation of In atoms to sites where they exhibit reduced $\mathrm{N}$ coordination, i.e. around the edges of the vacancy islands. This idea was tested on a structure consisting of an array of trenches. As shown in Figure 15(c), the trenches in this model system are created by removal of rows of layer 3 $\mathrm{N}$-atoms and layer $1 \mathrm{In}$-atoms. Total energy calculations employing a $6 \times 1$ unit cell to model such an array of trenches indicate that trench formation is exothermic and leads to strain relief [51]. It was concluded that this type of structural instability, in which surface strain is relieved and In segregates to the edges of the vacancy islands, is the fundamental mechanism giving rise to the vacancy islands.

During MBE growth of GaN, the surface undergoes a transition from smooth to rough morphology when the growth condition is switched from Ga-rich to $\mathrm{N}$-rich, as discussed below in Section 4. It was reported by Widmann et al. for the (0001) surface that indium atoms serve as a surfactant, keeping the growth in the smooth regime when the gallium flux is slightly reduced beneath the transition flux [54]. This surfactant effect was studied by Chen et al. in a study where they compared the influence of In on the kinetics for both the (0001) and $(000 \overline{1})$ faces [52]. A dramatic difference in the smooth/ rough behavior between the two faces was found, as shown in Figure 16. For these experiments, the nitrogen flux was kept constant. Then, a certain indium flux was applied, and gallium flux was adjusted to find the smooth/rough transition point. For comparison, dashed lines in Figure 16 show where the total metal flux (indium + gallium) is constant. Figure 16(a) shows that for the $(000 \overline{1})$ face, even when a large indium flux is applied, the gallium flux can only be reduced slightly before the growth becomes rough. In contrast, for the (0001) face, it is found that when the indium flux is applied the gallium flux can be greatly reduced (by an amount considerably greater than that of the added indium flux) before the growth becomes rough. Thus, indium serves as a surfactant for the (0001) surface but not for the $(000 \overline{1})$ surface. The interpretation of these observations is discussed below in Section 4 .

\subsection{Magnesium}

Magnesium is an important p-type dopant in GaN. To understand $\mathrm{Mg}$ incorporation kinetics during growth, a determination of the $\mathrm{Mg}$-induced surface reconstructions is required. In MBE growth of p-doped GaN, the high vapor pressure of $\mathrm{Mg}$ at $\mathrm{GaN}$ growth temperatures is an issue and dopant incorporation may be rather inefficient [55] [56]. Studies have shown that the $\mathrm{Mg}$ concentration decreases from the surface to the interior of the film [57], suggesting dopant incorporation from a surface $\mathrm{Mg}$ layer. Some workers have noted $\mathrm{Mg}$-induced changes in the growth rate of $\mathrm{GaN}$ on different crystallographic planes [58], pointing to a surfactant effect of $\mathrm{Mg}$ on $\mathrm{GaN}$. The presence of $\mathrm{Mg}$ atoms during the growth of $\mathrm{GaN}$ has also been associated with the appearance of stacking faults [59].

The surface science of $\mathrm{Mg}$ adlayers on the $\mathrm{GaN}(0001)$ surface has been studied by Ramachandran et al. using RHEED and STM [60] [61]. During PAMBE growth, films are briefly exposed to a Mg flux. A surfactant effect of $\mathrm{Mg}$ is seen on the Ga-polar films in the Ga-poor regime, where reducing the Ga-flux in the absence of Mg causes the RHEED pattern to change from streaky to spotty indicative of a growth mode transition from 2-dimensional to 3-dimensional [38]. Exposing this surface to about $0.2 \mathrm{ML}$ of $\mathrm{Mg}$ under $\mathrm{Ga}-$ poor conditions leads to a reversal of the RHEED pattern to streaky. Also, when the growth is made severely $\mathrm{N}$-rich, by reducing the Ga flux to about one half of that at the transition point, exposure to $\mathrm{Mg}$ often produces a streaky $2 \times 2$ pattern, as shown in Figure 17 . The origin of this surfactant behavior of $\mathrm{Mg}$ has not been considered in detail, although a model has been suggested in which $1 / 4$ or 3/4 ML of $\mathrm{Mg}$ substitutes for $\mathrm{Ga}$ in a $\mathrm{GaN}$ bilayer [56] [61], yielding a surface which satisfies electron counting and thus may produce reduced barriers for surface diffusion.

When the film is exposed to $1.2 \pm 0.4 \mathrm{ML}$ or more of $\mathrm{Mg}$ during growth, it is found that the polarity switches to $\mathrm{N}$-polar. After terminating the growth and cooling the sample to below about $300^{\circ} \mathrm{C}$, a $1 \times 1$ RHEED pattern is obtained. Exposing this surface to Ga produces $3 \times 3$, $6 \times 6$, and $c(6 \times 12)$ surfaces with increasing Ga coverage, as shown in Figure 17. This sequence of RHEED patterns definitively indicates the N-polarity of the film. Transmission electron microscopy (TEM) results reveal an inversion domain boundary extending along the cplane, as shown in Figure 18 [60]. The inverted film was found to contain numerous defects, perhaps arising from small inversion domains. More recent results from Romano et al. reveal an inverted film with fewer defects, 
and in that case the inversion boundary occurs on facetted planes [62].

To understand the origin of the polarity inversion, first principles pseudopotential calculations were performed of the total energy of various possible inverted structures. An IDB may form if, for a Mg concentration above a certain threshold, it is energetically favorable to

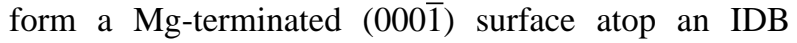
instead of a Mg-terminated (0001) surface. One such structure which was found is shown in Figure 19(a) [60]. In this case the IDB consists of a plane of Ga-Ga bonds and the surface is terminated by a monolayer of $\mathrm{Mg}$ in $\mathrm{H} 3$ sites. The $\mathrm{N}$ atoms in the outermost layer are six-fold coordinated with a local structure that is more like that of bulk $\mathrm{Mg}_{3} \mathrm{~N}_{2}$ than bulk GaN. It is found that this inverted structure is more stable (by $0.05 \mathrm{eV} / 1 \times 1$ cell) than the non-inverted, stoichiometrically identical, structure shown in Figure 19(b). It was conjectured that the inverted structure forms the template for subsequent growth of GaN, with the IDB frozen in place as a thicker $\mathrm{N}$-face film grows on top. It may also be possible that a few layers of bulk $\mathrm{Mg}_{3} \mathrm{~N}_{2}$ form above the IDB. The role of $\mathrm{Mg}$ in the formation of inversion domain boundaries on the facetted planes is discussed in Ref. [62].

\subsection{Arsenic}

As discussed above, the role of arsenic on the surface of $\mathrm{GaN}$ is controversial. A number of workers have reported an intense $2 \times 2$ diffraction observed during and after PAMBE growth on Ga-polar surfaces, and they interpret that in terms of a $2 \times 2$ arrangement of Ga adatoms. However, Ramachandran et al. [19] have presented convincing evidence that this structure arises, in fact, from arsenic atoms which are unintentionally present in the MBE growth systems. The presence of the arsenic may actually be beneficial for growth (it has a surfactant effect, described below), although the possibility remains of point defects arising from arsenic incorporation.

To understand the effect of arsenic, it is necessary to first describe the RHEED patterns observed on bare $\mathrm{GaN}(0001)$. During PAMBE growth under Ga-rich conditions, the RHEED pattern is generally $1 \times 1$ (occasionally $5 \times 5$ can be seen) and streaky, indicating smooth growth. If one changes the $\mathrm{Ga} / \mathrm{N}$ flux ratio so that $\mathrm{N}$-rich conditions are obtained, the pattern quickly changes to spotty, indicating rough growth, as shown in Figure 20(a)-(c). This smooth/rough transition is a hallmark of PAMBE growth, and its origin is discussed below in Section 4.

The effect of arsenic on the RHEED patterns during growth is dramatic, as shown in Figure 20(d)-(f) [19]. Arsenic was introduced by resistive heating of a GaAs wafer located in the growth chamber about $5 \mathrm{~cm}$ from the substrate holder. For arsenic beam equivalent pressure (BEP) below $1 \times 10^{-9}$ Torr, no change is detected in the RHEED sequence mentioned above. However, for higher arsenic BEP, as the Ga flux is reduced, a bright streaky $2 \times 2$ RHEED pattern is observed as shown in Figure $20(\mathrm{e})$. This transition to a $2 \times 2$ pattern is reversible, and it provides an unambiguous signature of the surface arsenic. Upon cooling, the $2 \times 2$ surface persists down to room temperature. Subsequent mild annealing of such a surface can cause the $2 \times 2$ pattern to switch to $4 \times 4$ [19]. This same $2 \times 2 / 4 \times 4$ behavior was reported by previous workers (who did not attribute the patterns to arsenic [37] [39]), and provides evidence that the $2 \times 2$ and $4 \times 4$ patterns they observe are indeed due to the presence of arsenic on the surface.

The streaky $2 \times 2$ pattern, occurring for a $\mathrm{Ga} / \mathrm{N}$ flux ratio near unit, demonstrates a surfactant nature of the arsenic adatoms. The window for this streaky $2 \times 2$ pattern in terms of fluxes is relatively broad and easily achieved during PAMBE growth. In contrast, if one wants to grow precisely at the $\mathrm{Ga} / \mathrm{N}=1$ point in the absence of arsenic (where the $1 \times 1$ RHEED pattern shows some brightening), the growth window is vanishingly small. Concerning the structure of the arsenicinduced $2 \times 2$, it seems likely that it arises simply from a $2 \times 2$ arrangement of arsenic adatoms which is a very low energy surface as described below. This surface satisfies electron counting (i.e. anion dangling bonds are doubly occupied and cation dangling bonds are empty [33]), and thus, qualitatively, it is expected to have relatively low diffusion barriers which may account for its surfactant behavior.

Possible models for the As-induced $2 \times 2$ structure include the adatom model as well as the As-trimer structure [19]. In both structures As atoms are bonded to Ga atoms in the layers below. A comparison of the stability of the possible structures as a function of the As chemical potential can be made and indicates that both adatom and trimer models can be stable with respect to the clean surfaces, depending on the chemical potentials of As and Ga. The As-adatom structure is preferred over the As-trimer structure for low values of the As chemical potential. Specifically, when $\mu_{\mathrm{As}}<\mu_{\mathrm{As} \text { (bulk) }}-0.49 \mathrm{eV}$ the As-adatom model is favored. For higher values of the chemical potential the As-trimer structure would be preferred. Because the surface energies of the Ga-rich surfaces are lower than those for N-rich conditions, the formation of As-terminated surfaces is more likely to occur under N-rich conditions. An analysis of the relative stability of As-terminated surfaces compared to clean surfaces [19] indicates that As-terminated surfaces are likely to be more stable than clean GaN sur- 
faces under the As deposition conditions employed in the experiments discussed above.

\subsection{Silicon}

\subsubsection{Experiment}

Silicon is commonly used as a n-type dopant in GaN. As in the case of $\mathrm{Mg}$ discussed above, aspects of its surface science can determine limits on the incorporation efficiency and structural quality of the resulting films. In addition, it has been shown that silicon has a strong effect on the surface morphology of GaN films: small amounts of silicon on GaN modify the growth mode from step-flow to 3-dimensional giving rise to the formation of small islands in MOVPE and gas source molecular beam epitaxy (GSMBE) [63] [64].

Lee et al. studied the effect of silicon adlayers on the surface structures of GaN(0001) surfaces [65]. Depositing $\mathrm{Si}$ on a Ga-rich (0001) surface, displaying a "1×1" reconstruction in RHEED, resulted in no change in the surface structure. The Si appears not to have modified the surface structure, as further discussed below. If, alternatively, $\mathrm{Si}$ is deposited on a (0001) surface displaying a $5 \times 5$ reconstruction, a $\mathrm{Si}$-induced $2 \times 2$ reconstruction results. Figure 21(a) shows a STM image of neighboring areas of the $2 \times 2$ and $5 \times 5$ reconstructions. Silicon exposure was performed at a temperature near $300^{\circ} \mathrm{C}$. With sufficient silicon exposure, a $2 \times 2$ pattern appears gradually. The temperature window for formation of the $2 \times 2$ reconstruction is quite narrow. With increasing substrate temperature the $2 \times 2$ disappears after it has formed, implying that it is metastable, and for lower temperatures no ordered surface structure is found. AES indicates a saturated $2 \times 2$ intensity for a $\mathrm{Si}$ coverage of roughly $0.5 \mathrm{ML}$.

Surfaces containing the Si-induced $2 \times 2$ structure also invariably display small regions of " $1 \times 1$ " structure. As the amount of $2 \times 2$ structure increases (due to additional $\mathrm{Si}$ deposition), the total area " $1 \times 1$ " regions also increases. When additional $\mathrm{Si}$, above $\approx 0.5 \mathrm{ML}$, is deposited on the surface, the $1 / 2$-order diffraction lines seen in RHEED become dim. The resulting surface appears in STM to be disordered, with small domains of wellordered $2 \times 2$ reconstructions surrounded by disordered regions, and containing as well numerous islands with " $1 \times 1$ " reconstruction.

Upon continuing the silicon exposure up to $\approx 1 \mathrm{ML}$ at $300^{\circ} \mathrm{C}$, the $2 \times 2$ reconstruction becomes weak and a new $4 \times 4$ pattern appears. This RHEED pattern is diffuse, indicating some surface disorder. In addition to $4 \times 4$, RHEED also shows a weak " $1 \times 1$ " pattern at room temperature. After annealing at around $350^{\circ} \mathrm{C}$ for 2 minutes, RHEED shows a clear $4 \times 4$ reconstruction. A large scale STM image for this sample is shown in Figure $22(a)$, and a detailed view of the $4 \times 4$ is shown in Figure 22(b). As seen there, the featureless " $1 \times 1 "$ region is dominant and the $4 \times 4$ region is seen only near step edges. With increasing anneal temperature, the $4 \times 4$ RHEED pattern disappears completely, and at room temperature only the " $1 \times 1 "$ pattern is seen. This indicates that the whole surface is covered by $\approx 2 \mathrm{ML} \mathrm{Ga}$ and the silicon atoms have moved to subsurface sites. Thus, based on these experimental observations it was concluded that the silicon adatoms tend to reside in subsurface sites on the Ga-polar surface.

\subsubsection{Theory}

In order to identify the atomic structure of the $\mathrm{Si}$ induced reconstructions density-functional theory calculations for a large number of possible geometries had been employed [66]. Specifically, starting from the known structures for bare $\mathrm{GaN}(0001) \mathrm{Si}$ atoms were systematically added on top of the surface or replaced $\mathrm{Ga} / \mathrm{N}$ atoms in the first, second and third layer. Si coverages $0<\Theta_{\mathrm{Si}}<2 \mathrm{ML}$ have been considered. To determine the stability of the different configurations the surface energy had been calculated as function of nitrogen $\left(\mu_{N}\right)$ and silicon $\left(\mu_{\mathrm{Si}}\right)$ chemical potential. Using these results a surface phase diagram had been derived which shows what surface is stable for a given set of chemical potentials (see Figure 23). The corresponding surface structures are shown in Figure 24. At low Si concentrations the incorporation of $\mathrm{Si}$ atoms into the surface is energetically unfavorable and only bare GaN surfaces are stable. Indeed going from $\mathrm{Ga}$ to $\mathrm{N}$ rich conditions the phase diagram reproduces the above described bare GaN surface structures (Ga-bilayer, Ga-adatom, N-adatom). When going towards more Si-rich conditions (close to the formation of Si-precipitates, i.e., $\mu_{\mathrm{Si}} \approx \mu_{\mathrm{Si}(\text { bulk) }}$ ) a number of Si-induced reconstructions are found. At Ga-rich conditions the Ga-bilayer structure with a Si in the third layer (Figure 24(c)) is energetically preferred. Going towards more N-rich conditions a structure with $1 \mathrm{ML}$ of $\mathrm{Si}$ is energetically most favorable (Figure 24(a)). At extreme $\mathrm{N}$ and $\mathrm{Si}$ rich conditions a structure with two $\mathrm{Si}$ and $\mathrm{N}$ layers is energetically favorable (Figure 24(g)). An important result of the phase diagram is that all $\mathrm{Si}$ induced surface reconstructions are thermodynamically unstable against the formation of $\mathrm{Si}_{3} \mathrm{~N}_{4}$.

Based on these calculations, the structures and structural changes observed in STM have been explained as follows. If $\mathrm{Si}$ adsorbs on the surface it kicks out surface $\mathrm{Ga}$ atoms and induces a $2 \times 2$ reconstruction (Figure 24(f); experiment: Figure 21). It is important to note that this Si induced $2 \times 2$ structure is metastable and therefore does not appear in the phase diagram. The metastability of the $2 \times 2$ structure is also found experimentally: The structure disappears if the sample is annealed above 
$350^{\circ} \mathrm{C}$. To create the $2 \times 2$ structure two Ga atoms per $2 \times 2$ cell have to be kicked out. These excess Ga atoms cluster in islands and increase locally the Ga-chemical potential. Based on the phase diagram one finds that under these conditions a Ga-bilayer with a pseudo- $1 \times 1$ structure stabilized by $\mathrm{Si}$ in the third layer is most stable. With increasing Si coverage more and more excess $\mathrm{Ga}$ atoms are created and the area covered by the Ga-bilayer will increase until eventually it covers the entire surface (as in Figure 22).

From the phase diagram trends concerning the incorporation of Si on GaN surfaces have also been deduced. For more N-rich conditions surface reconstructions with high Si-concentrations in the surface layer are found. Therefore, under those conditions, the Si concentration at the surface may be significantly higher than in GaN bulk and surface segregation might be an important issue. For more Ga-rich conditions, however, a fundamentally different behavior can be deduced from the phase diagram: Under these conditions Si prefers subsurface configurations rather than surface sites, i.e., surface segregation does not occur and Si can be efficiently incorporated in $\mathrm{GaN}$ bulk.

The above discussion gives also insight why in some experiments (employing MOVPE or GSMBE [63] [64]) $\mathrm{Si}$ acts as antisurfactant and roughens the surface while in others (PAMBE) it does not have this effect. The main difference between the two cases is that in MOVPE and GSMBE growth hydrogen is highly abundant while in PAMBE it is virtually absent. As will be shown in the next Section, hydrogen stabilizes $\mathrm{N}$ on the surface making the surface more N-rich. The structures found under these conditions (Figs. 24(a) and (g)) have in the top surface layers exclusively $\mathrm{Si}$ and $\mathrm{N}$ atoms and the activation barrier to form $\mathrm{Si}_{3} \mathrm{~N}_{4}$ is thus expected to be rather low. In fact, calculations for an isolated $\mathrm{Si}-\mathrm{N}$ double layer indicate that the surface layer of these structures is rather unstable and under large tensile strain: Going from an in-plane lattice constant of $3.19 \AA$ for $\mathrm{GaN}$ to the smaller value of $2.86 \AA$ leads to a reduction in energy of $0.92 \mathrm{eV}$ per $\mathrm{N}$ atom. The formation of $\mathrm{Si}_{3} \mathrm{~N}_{4}$ has important consequences. On one side, it explains the antisurfactant behavior of $\mathrm{Si}$ on $\mathrm{GaN}(0001)$. $\mathrm{Si}_{3} \mathrm{~N}_{4}$ islands/precipitates are well known to chemically passivate the GaN surface and to block growth [67]. Since growth occurs then only on areas not covered by $\mathrm{Si}_{3} \mathrm{~N}_{4}$ three-dimensional growth results.

Under Ga-rich conditions (which are characteristic for PAMBE growth), the Si induced surfaces are essentially free of $\mathrm{Si}$ in the top surface layer. Since for these structures $\mathrm{Si}$ is already incorporated in a bulk-like GaN environment the formation of $\mathrm{Si}_{3} \mathrm{~N}_{4}$ is expected to be largely suppressed by kinetic barriers. Further, since the topology of these surfaces is very similar to the bare surfaces $\mathrm{Si}$ has no effect on the adatom kinetics or the growth mode. Ga-rich conditions are therefore expected to be the optimum regime to incorporate $\mathrm{Si}$ in $\mathrm{GaN}$.

\subsection{Hydrogen}

Hydrogen is present in high concentrations in commonly used growth techniques for nitride semiconductors, including MOVPE, hydride vapor-phase epitaxy (HVPE) and GSMBE (using an $\mathrm{NH}_{3}$ source). Hydrogen has been observed to have important effects on the growth of GaN. For example, Yu et al. [68] observed that the introduction of $\mathrm{H}$ during $\mathrm{MBE}$ growth of $\mathrm{GaN}$ using an RF-plasma source can increase the growth rate by as much as a factor of two. Hydrogen has been also found to improve the quality of GaN in PAMBE [69].

Experimental investigations of how hydrogen modifies the GaN surfaces and surface kinetics are rare. Sung et al. [20] investigated the composition and structure of $\mathrm{GaN}(000 \overline{1})$ surfaces grown by MOVPE using time-offlight scattering and recoiling spectrometry, LEED, and thermal decomposition mass spectrometry. Based on these studies they concluded that hydrogen is on the surface, that it removes surface states, and facilitates autocompensation (i.e. partially occupied dangling bonds are passivated by hydrogen). Hydrogen desorption was studied by several groups [70] [71]. Bellitto et al. [71] demonstrated by high resolution electron energy loss spectroscopy, electron energy loss spectroscopy, and LEED that hydrogen desorption from $\mathrm{Ga}$ sites occurs between 250 and $450^{\circ} \mathrm{C}$. Vibrational modes of hydrogen on $\mathrm{GaN}$ have been observed by several groups. Bellitto et al. [71] and Grabowski et al. [72] observed Ga-H modes at $1880 \mathrm{~cm}^{-1}$ and $1900 \mathrm{~cm}^{-1}$, respectively. $\mathrm{N}-\mathrm{H}$ modes where found at $3255 \mathrm{~cm}^{-1}$ [70].

All of the above described studies were performed far away from realistic growth conditions. Using thermal desorption mass spectrometry Held et al. [73] determined surface reactivity and growth kinetics during RMBE growth. A model for the observations was developed involving multiple adsorption states for $\mathrm{Ga}$ on the surface. Based on the structural studies of Smith et al. [30] discussed above the origin of these adsorption states can be identified. Additional models for surface kinetics during RMBE growth have also been reported [12] [74] [75]. Those models assume, in general, the existence of surface layers of particular types in order to match the observed kinetic data, thereby gaining valuable insight concerning the composition of the surface layers. Nevertheless, it should be noted that the detailed structures and composition of such surface layers for RMBE are not yet well understood on the basis of independent surface structural studies. 
Using grazing incidence $\mathrm{x}$-ray scattering Munkholm et al. [14] performed in situ studies of surface reconstructions on $\mathrm{GaN}(0001)$ surfaces in an MOVPE environment. In that study it was found that the surface equilibrium phase diagram as a function of temperature and ammonia partial pressure shows a transition between two phases: at high temperatures, a $1 \times 1$ reconstruction was observed, while at lower temperatures and sufficiently low $\mathrm{NH}_{3}$ pressures, a different reconstruction with $2 \sqrt{ } 3 \times 2 \sqrt{ } 3-\mathrm{R} 30^{\circ}$ periodicity was seen. From the temperature dependence of $p\left(\mathrm{NH}_{3}\right)$ at the transition, an activation energy of $3.0 \pm 0.2 \mathrm{eV}$ was extracted.

Early theoretical studies of $\mathrm{H}$ on $\mathrm{GaN}(0001)$ and/or $(000 \overline{1})$ surfaces gave important insight but were restricted on a few selected structures and did not include temperature and pressure dependence [76] [77] [78]. A main conclusion from these studies was that $\mathrm{H}$ strongly binds to GaN surfaces. Further studies by Northrup et al. [79] for GaN(1100) and by Van de Walle et al. [80] however showed that at typical MOVPE or HVPE conditions $\left(T=1050^{\circ} \mathrm{C}, p \sim 1 \mathrm{~atm}\right)$ hydrogen is only weakly bound to the surface and affects the surface energy only slightly.

Van de Walle et al. [80] investigated over 30 different surface reconstructions of hydrogenated $\mathrm{GaN}(0001)$ including structures with $1 \times 1,2 \times 2$ and $\sqrt{3} \times \sqrt{3}$ periodicity. An important conclusion of this study was that only structures obeying the electron counting rule are energetically favorable, i.e. hydrogen efficiently removes surface states and compensates partially occupied surface states. All hydrogen terminated surfaces are therefore semiconducting, in contrast to bare $\mathrm{GaN}(0001)$ where metallic structures (such as the Ga bilayer [33]) are found. Based on the calculated surface energies a surface phase diagram (Figure 25) has been constructed that shows which surface is energetically most stable for a given set of $\mathrm{Ga}$ and $\mathrm{H}$ chemical potentials. The corresponding reconstructions are shown in Figure 26. For $\mathrm{H}$ poor conditions $\left(\right.$ low $\mu_{\mathrm{H}}$ ) the phase diagram reproduces the reconstructions as calculated [30] [46] for bare $\mathrm{GaN}(0001)$ : Going from N-rich to Ga-rich conditions a $\mathrm{N}$ adatom, a $\mathrm{Ga}$ adatom and a Ga-bilayer structure are most stable. In the presence of hydrogen the structure most favorable at zero temperature $\left(\mu_{\mathrm{H}}=0 \mathrm{eV}\right)$ is the $\mathrm{NH}_{3}+3 \mathrm{NH}_{2}$ reconstruction. In this structure one $\mathrm{NH}_{3}$ and three $\mathrm{NH}_{2}$ molecules are attached on the Ga-terminated surface in on-top positions (i.e. with the $\mathrm{N}$ atoms of the molecules above the Ga surface atoms). It can thus be concluded that at low temperatures $\mathrm{NH}_{3} / \mathrm{NH}_{2}$ molecules are thermodynamically stable - dissociation of these molecules is forbidden. Going towards less hydrogen rich conditions not all the $\mathrm{N}$ atoms can be kept at the surface and $\mathrm{Ga}-\mathrm{H}$ bonds are formed. Examples are the $\mathrm{NH}_{3}+3 \mathrm{Ga}-\mathrm{H}$ structure (where compared to the $\mathrm{NH}_{3}+3 \mathrm{NH}_{2}$ surface the $3 \mathrm{NH}_{2}$ molecules have been replaced by $\mathrm{H}$ atoms), the $3 \mathrm{Ga}-\mathrm{H}$ structure (where all $\mathrm{NH}_{3} / \mathrm{NH}_{2}$ have been removed), or the $\mathrm{N}_{\mathrm{ad}}-\mathrm{H}+\mathrm{Ga}-\mathrm{H}$ structure (where only a single $\mathrm{NH}$ group remains). An important conclusion that can be drawn from these results is that dissociation of the $\mathrm{NH}_{3}$ molecule is only possible at sufficiently low hydrogen chemical potential and that the tendency to dissociate is strongly enhanced when going towards Ga-rich conditions.

Van de Walle et al. [80] also give an explicit dependence of the hydrogen chemical potential on temperature and pressure which allows to relate experimental growth conditions to surface structures in the phase diagram. For realistic growth conditions (between 700 and $1100^{\circ} \mathrm{C}$ ) and not too $\mathrm{N}$-rich conditions surfaces with only $\mathrm{NH}_{3} / \mathrm{NH}_{2}$ molecules are unstable, i.e., a dissociation of ammonia molecules is thermodynamically favorable. The explicit knowledge of the hydrogen chemical potential made it also possible to include in the phase diagram the above mentioned and thus far only experimentally observed transition between two surface reconstructions (see the dotted line in Figure 25) [14]. As can be seen the experimental data are (within the estimated error bar of $0.1 \mathrm{eV}$ ) consistent with a transition between the $\mathrm{NH}_{3}+3 \mathrm{Ga}-\mathrm{H}$ and the $3 \mathrm{Ga}-\mathrm{H}$ structure, i.e., with adding/removing a $\mathrm{NH}_{3}$ molecule.

\section{Surface Kinetics}

A major goal of the surface science studies presented above is to serve as a basis for developing an understanding of surface kinetics. With the detailed knowledge of surface structures gained over the past number of years, realistic models of kinetic processes are now becoming possible. Several such studies of kinetics during RMBE were mentioned in the previous Section on hydrogen adsorbates. For the case of PAMBE of GaN, as discussed above in Section 3.1, a hallmark of that growth process is a smooth to rough transition which occurs with decreasing Ga flux [38] [81] [82] [83] [84]. This behavior is illustrated in Figure 27. Similar behavior occurs for both $(000 \overline{1})$ and (0001) surfaces. The transition from rough to smooth morphology occurs when the Ga flux exceeds some critical flux, which itself scales with the incident $\mathrm{N}$ flux. (The absolute $\mathrm{Ga}$ to $\mathrm{N}$ flux ratio is generally not quoted due to difficulties in measuring the active $\mathrm{N}$ flux, but often the flux ratio is simply defined to be unity at the transition point [38]). Given this smooth/rough growth behavior, PAMBE growth is nearly always performed under Ga-rich conditions. For very Ga-rich conditions $\mathrm{Ga}$ droplets are observed to form on the surface, whereas for less Garich conditions pits form on the surface in the vicinity of 
threading dislocations [82] [83] [84]. Achieving an operating point between these regimes is generally believed to provide an optimal growth condition.

The origin of this smooth to rough behavior of the GaN surface has been discussed by Zywietz et al. [85]. They computed surface diffusion barriers for Ga- and Nadatoms on Ga-terminated (0001) and (000) $)$ surfaces, finding much larger barriers for the $\mathrm{N}$-adatoms on both surfaces. It was argued that this limited diffusivity of the $\mathrm{N}$-adatoms will lead, under growth conditions, to a significant surface coverage of $\mathrm{N}$ (although in general one does not expect a significant coverage of $\mathrm{N}$ atoms on the surface since the stable reconstructions are almost all terminated by $\mathrm{Ga}$ atoms, the $\mathrm{N}$ adatoms may kinetically accumulate during growth under $\mathrm{N}$-rich conditions). It was furthermore found that on N-terminated surfaces, the diffusion barrier for $\mathrm{Ga}$ adatoms increases to about 1.8 or $1.0 \mathrm{eV}$ for (0001) or (000) $)$ surface respectively. These barriers are believed to be large enough to produce a rough growth morphology.

An alternative model for the smooth to rough behavior has been suggested by Chen et al. [52] based on kinetic effects in the presence of indium. As described above in Section 3.1, the addition of indium to the surface produces much different behavior of the smooth to rough transition for $(000 \overline{1})$ and (0001) surfaces. In the former case the transition point (i.e. flux ratio at which the transition occurs) is nearly unchanged as additional indium is added, whereas in the latter case the addition of only a small amount of indium leads immediately to smooth growth even under $\mathrm{N}$-rich conditions. This behavior has been correlated with the amount of metal (In or $\mathrm{Ga}$ ) on the surface. Under N-rich conditions, the $\mathrm{N}$-polar surface is terminated by only $1 \mathrm{ML}$ of metal, with this metal layer being bonded to underlying $\mathrm{N}$ atoms. $\mathrm{N}$-adatoms are expected to diffuse on top of this metal layer, with relatively large diffusion barriers. In contrast, on the Ga-polar surface there are two layers of metal even under $\mathrm{N}$-rich conditions. $\mathrm{N}$-adatoms can then diffuse in between these metal layers. The top metal layer is bonded with metallic bonds to underlying metal atoms and is thus relatively easy to move. The double layer of metal can thus rearrange to accommodate the $\mathrm{N}$ atoms, thereby producing substantially reduced diffusion barriers for the $\mathrm{N}$.

This model of enhanced $\mathrm{N}$ diffusivity in the presence of multiple metal layers also is applicable to the case of bare $\mathrm{GaN}$ growth, under Ga-rich conditions. Indeed, Ga has been argued to provide a surfactant effect for the growth of $\mathrm{GaN}$ [43]. For both the (0001) and (0001) surface, there are excess metal atoms on the surface, and these atoms may act to provide greater coordination to diffusing $\mathrm{N}$ adatoms, thereby enhancing their diffusivity. It should be noted that the two models described above - reduced Ga diffusivity in the presence of an accumulation of $\mathrm{N}$ (N-rich conditions) or enhanced $\mathrm{N}$ diffusivity in the presence of excess $\mathrm{Ga}$ (Ga-rich conditions) - both act in the same direction to produce the observed smooth/rough behavior of the surface morphology. Indeed, since excess $\mathrm{Ga}$ atoms present under Ga-rich conditions will necessarily act to suppress any accumulation of $\mathrm{N}$-atoms, it is not completely clear whether both models are required to produce the observed behavior. Further study is required to allow discrimination of the various kinetic mechanisms.

Growth at reduced temperature is a commonly used technique in the study of growth kinetics. This method has been used by Zheng et al. to elucidate some kinetic aspects of GaN growth [86]. Using PAMBE, they deposit $\mathrm{GaN}$ on $\mathrm{GaN}(0001)$ surfaces at temperatures of about $400^{\circ} \mathrm{C}$. Small, isolated islands are formed on the surface (at higher growth temperature, the growth proceeds by step flow so that islands are not formed). It is found that two types of islands form: "normal" islands which display a regular bilayer $(2.6 \AA)$ step height, and "ghost" islands which in STM image display a distinct boundary around the island but have a island height much less than 1 bilayer. AFM studies revealed that the ghost islands do in fact have height of nearly 1 bilayer, so that their different appearance in STM images could be ascribed to electronic effects (i.e. implying different structure for those islands) [86]. It was noted that for ideal growth, $\mathrm{N}$ atoms should substitute for the $\mathrm{Ga}$ atoms in the second layer of the Ga double layer terminating the surface [86]. To achieve the different structure of the ghost islands, it was thus proposed that the Natoms substituted instead for Ga atoms in the first layer of the Ga double layer. The resulting structure would then have this N-layer bonded on top of two layers of $\mathrm{Ga}$ atoms (i.e. the lower layer of the double layer plus the Ga-layer of the uppermost GaN bilayer). These two $\mathrm{Ga}$ layers have a Ga-Ga bonding arrangement similar to that shown in Figure 19 above; this arrangement provides a relatively low energy structure. The ghost islands are thus seen to constitute regions of inverted polarity on the surface [86].

\section{Summary}

In summary, we have reviewed here most of the studies reported to date concerning the surface structures of $\mathrm{GaN}(0001)$ and (000) $)$ surfaces. Structures of the bare surfaces, as a function of surface stoichiometry, are now fairly well understood. The surface structures of InGaN are also largely understood, and good progress has also been made in determining the structure and energetics for adsorbed atoms of $\mathrm{Mg}, \mathrm{As}, \mathrm{Si}$ and $\mathrm{H}$. A defining feature of many of these surface structures, as they occur during PAMBE, is the occurrence of one or more layers 
of metal atoms on the surface. This is contrary to what occurs on most other semiconductor surfaces (where non-metallic surfaces generally have lower energy, i.e. due to the opening of a band gap), but it occurs in the case of $\mathrm{GaN}$ due in part to the large difference in size between $\mathrm{Ga}$ and $\mathrm{N}$ so that monolayers of $\mathrm{Ga}$ (or In) can more or less fit onto the GaN surface. These metallic layers then have great influence on the surface kinetics. An open question at this time is whether or not similar metallic layers also exist during growth by other methods such as MOVPE, RMBE or HVPE. Future work should lead to a greater understanding of the stable surface structures during growth by those methods, thus enabling a comparison of the relative efficacy of the different methods for processes such as alloy formation and dopant incorporation.

\section{ACKNOWLEDGMENTS}

We thank H. Chen, D. W. Greve, C. D. Lee, V. Ramachandran, A. L. Rosa, A. R. Smith, C. G. Van de Walle and T. Zywietz for their collaboration in the work described in part in this paper. We gratefully acknowledge support from the U. S. Office of Naval Research under Grant N00014-96-1-0214 (R.M.F.), from the U.S. Air Force Office of Scientific Research under Contract F4920-00-C-0019 (J.E.N.), and from the German Research Society (J.N.).

\section{REFERENCES}

[a] Indium exhibits a body-centered-tetragonal structure with each indium atom having 4 neighbors at $3.25 \AA$ and 8 neighbors at $3.38 \AA$. Gallium adopts an orthorhombic structure with each Ga atom having one neighbor at $2.47 \AA, 2$ at $2.70 \AA$, 2 at $2.73 \AA$ and 2 at $2.79 \AA$. The weighted average distances are $\sim 3.3 \AA$ for In and $\sim 2.7 \AA$ for Ga. The structures of indium and gallium are discussed in J. Donohoe, The Structure of the Elements (Robert E. Krieger Publishing Company, Malabar, Florida,1982).

[1] R. F. Davis, Proc. IEEE 79, 702 (1991).

[2] I Akasaki, H Amano, J. Electrochem. Soc. 141, 22662271 (1994).

[3] H. Morkoc, S. Strite, G. B. Gao, M. E. Lin, B. Sverdlov, M. Burns , J. Appl. Phys. 76, 1363-1398 (1994).

[4] S. Nakamura, 281, 956 (1998).

[5] N. M. Johnson, A. V. Nurmikko, S. P. DenBaars, Phys. Today 53, 31 (2000).

[6] S. C. Jain, M. Willander, J. Narayan, R. Van Overstraeten, J. Appl. Phys. 87, 965 (2000).

[7] D. Doppalapudi, T. D. Moustakas, "Epitaxial Growth and Structure of III-V Nitride Thin Films", Handbook of Thin Film Materials, ed. H. S. Nalwa, Volume 4: Semiconductor and Superconductor Thin Films (Academic Press, 2002)

[8] G. Feuillet, H. Hamaguchi, K. Ohta, P. Hacke, H. Okumura, S. Yoshida, Appl. Phys. Lett. 70, 1025-1027 (1997).

[9] M. Wassermeier, A. Yamada, H. Yang, O. Brandt, J. Behrend, K. H. Ploog, Surf. Sci. 385, 178 (1997).
[10] Joerg Neugebauer, Tosja K. Zywietz, Matthias Scheffler, John E. Northrup, Chris G. Van de Walle, Phys. Rev. Lett. 80, 3097 (1998).

[11] R. Held, G. Nowak, B.E. Ishaug, S.M. Seutter, A. Parkhomovsky, A.M. Dabiran, P.I. Cohen, I. Grzegory, S. Porowski, J. Appl. Phys. 85, 7697-7704 (1999).

[12] N. Grandjean, J. Massies, F. Semond, S. Yu. Karpov, R. A. Talalaev, Appl. Phys. Lett. 74, 1854 (1999).

[13] A. Thamm, O. Brandt, Y. Takemura, A. Trampert, K. H. Ploog, Appl. Phys. Lett. 75, 944 (1999).

[14] A. Munkholm, G. B. Stephenson, J. A. Eastman, C. Thompson, P. Fini, J. S. Speck, O. Auciello, P. H. Fuoss, S. P. DenBaars, Phys. Rev. Lett. 83, 741 (1999).

[15] E. S. Hellman, MRS Internet J. Nitride Semicond. Res. 3, 11 (1998).

[16] M.J. Murphy, B.E. Foutz, K. Chu, H. Wu, W. Yeo, W.J. Schaff, O. Ambacher, L.F. Eastman, T.J. Eustis, R. Dimitrov, M. Stutzmann, W. Rieger, MRS Internet J. Nitride Semicond. Res. 4S1, G8.4 (1999).

[17] M. Sumiya, T. Ohnishi, M. Tanaka, A. Ohtomo, M. Kawasaki, M. Yoshimoto, K. Koinuma, K. Ohtsuka, S. Fuke, MRS Internet J. Nitride Semicond. Res. 4S1, G6.23 (1999).

[18] V. Ramachandran, C. D. Lee, R. M. Feenstra, A. R. Smith, D. W. Greve, Phys. Rev. Lett. 84, 4014 (2000).

[19] V. Ramachandran, C. D. Lee, R. M. Feenstra, A. R. Smith, J. E. Northrup, D. W. Greve, J. Cryst. Growth 209, 355 (2000).

[20] M. M. Sung, J. Ahn, V. Bykov, J. W. Rabalais, D. D. Koleske, A. E. Wickenden, Phys. Rev. B 54, 14652-14663 (1996).

[21] J. Ahn, M. M. Sung, J. W. Rabalais, D. D. Koleske, A. E. Wickenden, J. Chem. Phys. 107, 9577-9584 (1997).

[22] M. Asif Khan, J. N. Kuznia, D. T. Olson, R. Kaplan , J. Appl. Phys. 73, 3108-3110 (1993).

[23] W. C. Hughes, W. H. Rowland, M. A. L. Johnson, Shizuo Fujita, J. W. Cook, J. F. Schetzina, J. Ren, J. A. Edmond, J. Vac. Sci. Technol. B 13, 1571-1577 (1995).

[24] M. E. Lin, S. Strite, A. Agarwal, A. Salvador, G. L. Zhou, N. Teraguchi, A. Rockett, H. Morkoc, Appl. Phys. Lett. 62, 702-704 (1993).

[25] K IWATA, H ASAHI, SJ YU, K ASAMI, H FUJITA, M FUSHIDA, S GONDA, Jpn. J. Appl. Phys. 35, L289 (1996).

[26] P Hacke, G Feuillet, H Okumura, S Yoshida, Appl. Phys. Lett. 69, 2507-2509 (1996).

[27] W. S. Wong, N. Y. Li, H. K. Dong, F. Deng, S. S. Lau, C. W. Tu, J. Hays, S. Bidnyk, J. J. Song, J. Cryst. Growth 164, 159 (1996).

[28] R.J. Molnar, R. Singh, T.D. Moustakas, J. Electron. Mater. 24, 275 (1995).

[29] W. E. Packard, J. D. Dow, R. Nicolaides, K. Doverspike, R. Kaplan, Superlatt. Microstruc. 20, 145 (1996).

[30] A. R. Smith, R. M. Feenstra, D. W. Greve, J. Neugebauer, J. E. Northrup, Phys. Rev. Lett. 79, 3934 (1997).

[31] A. R. Smith, R. M. Feenstra, D. W. Greve, J. Neugebauer, J. E. Northrup, Appl. Phys. A 66, S947 (1998).

[32] G. X. Qian, R. M. Martin, D. J. Chadi, Phys. Rev. B 38, 7649 (1988). 
[33] AR Smith, RM Feenstra, DW Greve, M-S Shin, M Skowronski, J Neugebauer, J Northrup, J. Vac. Sci. Technol. B 16, 2242-2249 (1998).

[34] Ryan, Y. C. Chao, J. Downes, C. McGuinness, K. E. Smith, A. V. Sampath, T. D. Moustakas, Surf. Sci. 467, L827 (2000).

[35] Huajie Chen, R. M. Feenstra, J. Northrup, Jörg Neugebauer, D. W. Greve, MRS Internet J. Nitride Semicond. Res. 6, 11 (2001).

[36] A. R. Smith, R. M. Feenstra, D. W. Greve, M. S. Shin, M. Skowronski, J. Neugebauer, J. E. Northrup, Surf. Sci. 423, 70 (1999).

[37] Q. K. Xue, Q. Z. Xue, R. Z. Bakhtizin, Y. Hasegawa, I. S. T. Tsong, T. Sakurai, T. Ohno, Phys. Rev. Lett. 82, 3074 (1999).

[38] A. R. Smith, V. Ramachandran, R. M. Feenstra, D. W. Greve, A. Ptak, T. Myers, W. Sarney, L. Salamanca-Riba, M. Shin, M. Skowronski, MRS Internet J. Nitride Semicond. Res. 3, 12 (1998).

[39] Q. Z. Xue, Q. K. Xue, S. Kuwano, J. T. Sadowski, K. F. Kelly, T. Sakurai, T. Ohno, Phys. Rev. Lett. 84, 4015 (2000).

[40] O. Brandt, private communication

[41] D. L. Abernathy, D. Gibbs, G. Gruebel, K. G. Huang, S. G. J. Mochrie, A. R. Sandy, D. M. Zehner, Surf. Sci. 283, 260 (1993).

[42] R. M. Feenstra, H. Chen, V. Ramachandran, A. R. Smith, D. W. Greve, Appl. Surf. Sci. 166, 165 (2000).

[43] G. Mula, C. Adelmann, S. Moehl, J. Oullier, B. Daudin, Phys. Rev. B 64, 195406 (2001).

[44] A. Pavlovska, E. Bauer, Surf. Sci. 480, 128 (2001).

[45] A. Pavlovska, E. Bauer, Surf. Rev. Lett. 8, 337 (2001).

[46] JE Northrup, J Neugebauer, RM Feenstra, AR Smith, Phys. Rev. B 61, $9932-9935$ (2000).

[47] S. Y. Tong, G. Xu, W. N. Mei, Phys. Rev. Lett. 52, 1693 (1984).

[48] D. J. Chadi, Phys. Rev. Lett. 52, 1911 (1984).

[49] J. E. Northrup, R. Di Felice, J. Neugebauer, Phys. Rev. B 55, 13878 (1997).

[50] Huajie Chen, A.R. Smith, R.M. Feenstra, D.W. Greve, J.E. Northrup, MRS Internet J. Nitride Semicond. Res. 4S1, G9.5 (1999).

[51] H Chen, RM Feenstra, JE Northrup, T Zywietz, J Neugebauer, Phys. Rev. Lett. 85, 1902-1905 (2000).

[52] H Chen, RM Feenstra, JE Northrup, T Zywietz, J Neugebauer, DW Greve, J. Vac. Sci. Technol. B 18, 2284-2289 (2000).

[53] JE Northrup, J Neugebauer, Phys. Rev. B 60, 8473-8476 (1999).

[54] Widmann, B. Daudin, G. Feuillet, J. L. Rouviére, N. Pelekanos, Appl. Phys. Lett. 73, 2642 (1998).

[55] S Guha, NA Bojarczuk, F Cardone, Appl. Phys. Lett. 71, 1685-1687 (1997).

[56] C. Bungaro, K. Rapcewicz, J. Bernholc, Phys. Rev. B 59, 9771 (1999).

[57] T. S. Cheng, C. T. Foxon, N. J. Jeffs, D. J. Dewsnip, L. Flannery, J. W. Orton, S. V. Novikov, B. Ya Ber, Yu A. Kudriavtsev, MRS Internet J. Nitride Semicond. Res. 2, 13 (1997).
[58] B. Beaumont, S; Haffouz, P. Gibart, Appl. Phys. Lett. 72, 921 (1998).

[59] Z. Lilienthal-Weber, M. Benamara, W. Swider, J. Washburn, Grzegory, S. Porowski, R. D. Dupuis, C. J. Eiting, Physica B 273-274, 124 (1999).

[60] V. Ramachandran, R. M. Feenstra, W. L. Sarney, L. Salamanca-Riba, J. E. Northrup, L. T. Romano, D. W. Greve, Appl. Phys. Lett. 75, 808 (1999).

[61] Vidhya Ramachandran, Randall M Feenstra, John E. Northrup, David W. Greve, MRS Internet J. Nitride Semicond. Res. 5S1, W3.65 (2000).

[62] L. T. Romano, J. E. Northrup, A. J. Ptak, T. H. Myers, Appl. Phys. Lett. 77, 2479 (2000).

[63] S Tanaka, S Iwai, Y Aoyagi, Appl. Phys. Lett. 69, 40964098 (1996).

[64] XQ Shen, S Tanaka, S Iwai, Y Aoyagi, Appl. Phys. Lett. 72, 344-346 (1998).

[65] C. D. Lee, R. M. Feenstra, A. L. Rosa, J. Neugebauer, J. E. Northrup, J. Vac. Sci. Technol. B 19, 1619 (2001).

[66] A. L. Rosa, J. Neugebauer, J. E. Northrup, C. D. Lee, R. M. Feenstra, Appl. Phys. Lett. 80, 2008 (2002).

[67] H. Lahreche, P. Vennegues, B. Beaumont, P. Gibart, J. Cryst. Growth 205, 245 (1999).

[68] Z Yu, SL Buczkowski, NC Giles, TH Myers, MR Richards-Babb, Appl. Phys. Lett. 69, 2731-2733 (1996).

[69] Y. Okamoto, S. Hashiguchi, Y. Okada, M. Kawabe, Jpn. J. Appl. Phys. 38, L230 (1999).

[70] C. M. Chiang, S. M. Gates, A. Bensaoula, J. A. Schultz, Chem. Phys. Lett. 246, 275 (1995).

[71] V. J. Bellito, Y. Yang, B. D. Thoms, D. D. Koleske, A. E. Wickenden, R. L. Henry, Surf. Sci. 442, L1019 (1999).

[72] S. P. Grabowski, H. Nienhaus, W. Moench, Surf. Sci. 454, 498 (2000).

[73] R. Held, B. E. Ishaug, A. Parkhomovsky, A. M. Dabiran, P. I. Cohen, J. Appl. Phys. 87, 1219 (2000).

[74] S. Y. Karpov, M. A. Maiorov, Surf. Sci. 393, 108 (1997).

[75] Wenning Fu, Rama Venkat, J. Vac. Sci. Technol. B 18, 1467 (2000).

[76] K. Rapcewicz, M. B. Nardelli, J. Bernholc, Phys. Rev. B 56, 12725-12728 (1997).

[77] J. Fritsch, O. F. Sankey, K. E. Schmidt, J. B. Page, Phys. Rev. B 57, 15360 (1998).

[78] J Elsner, M Haugk, G Jungnickel, Th Frauenheim, Sol. St. Comm. 106, 739-743 (1998).

[79] J. E. Northrup, R. Di Felice, J. Neugebauer, Phys. Rev. B 56, 4325 (1997).

[80] C. G. Van de Walle, J. Neugebauer, Phys. Rev. Lett. 88, 066103 (2002).

[81] EJ Tarsa, B Heying, XH Wu, P Fini, SP DenBaars, JS Speck, J. Appl. Phys. 82, 5472-5479 (1997).

[82] K. H. Ploog, O. Brandt, R. Muralidharan, A. Thamm, P. Waltereit, J. Vac. Sci. Technol. B 18, 2290 (2000).

[83] B. Heying, R. Averbeck, L. F. Chen, E. Haus, H. Riechert, J. S. Speck, J. Appl. Phys. 88, 1855 (2000).

[84] C. D. Lee, V. Ramachandran, A. Sagar, R. M. Feenstra, D. W. Greve, W. L. Sarney, L. Salamanca-Riba, D. C. Look, S. Bai, W. J. Choyke, R. P. Devaty, J. Electron. Mater. 30, 162 (2001). 
[85] T. K. Zywietz, J. Neugebauer, M. Scheffler, Appl. Phys. Lett. 73, 487 (1998).

[86] L. X. Zheng, M. H. Xie, S. M. Seutter, S. H. Cheung, S. Y. Tong, Phys. Rev. Lett. 85, 2352 (2000).
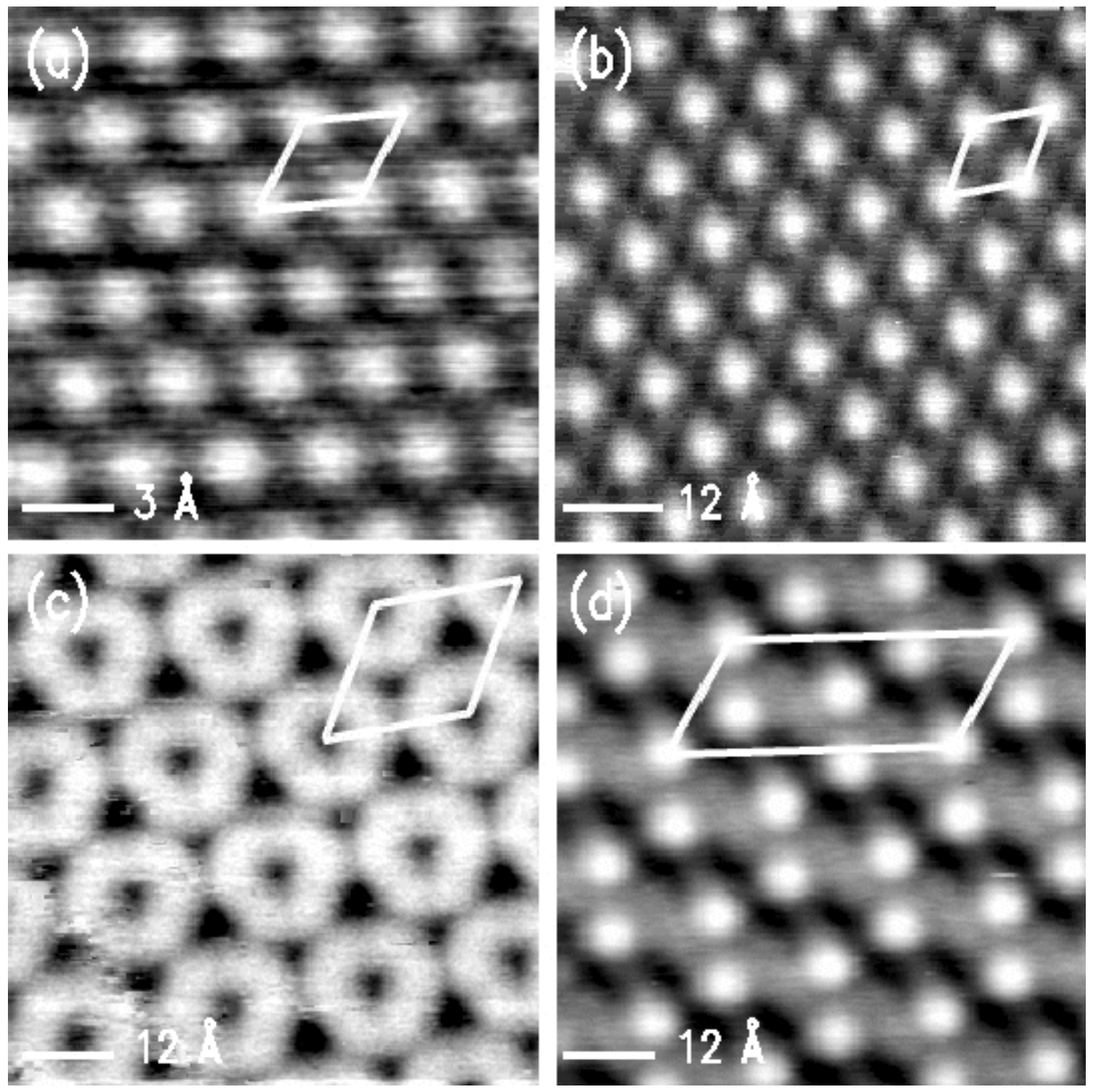

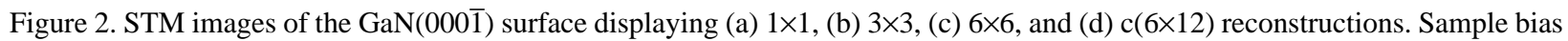
voltages are $-0.75,-0.1,+1.5$, and $+1.0 \mathrm{~V}$, respectively. Tunnel currents are in the range $0.03-0.11 \mathrm{nA}$. Gray scale ranges are 0.17 , 0.88, 1.33, and $1.11 \AA ̊$ A respectively. Unit cells are indicated with edges along <11 $\overline{2} 0>$ directions. (From [30]). 


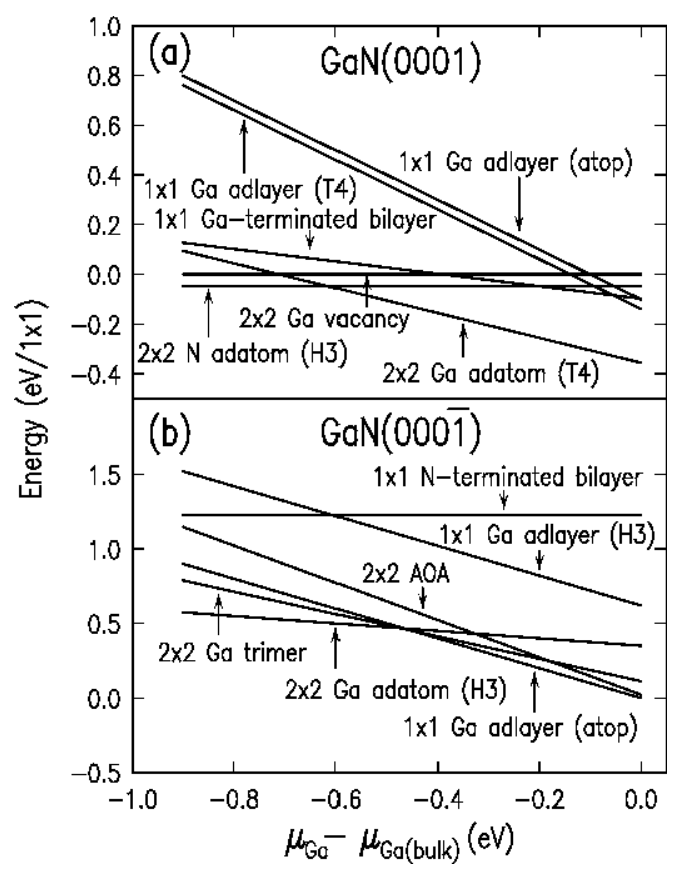

Figure 3. (a) The relative energies calculated for possible models of the $\mathrm{GaN}(0001)$ surface are shown as a function of the Ga chemical potential. (b) Relative energies for $\mathrm{GaN}(000 \overline{1})$ surfaces. The zeroes of energy in (a) and (b) are not related. (From [30]).

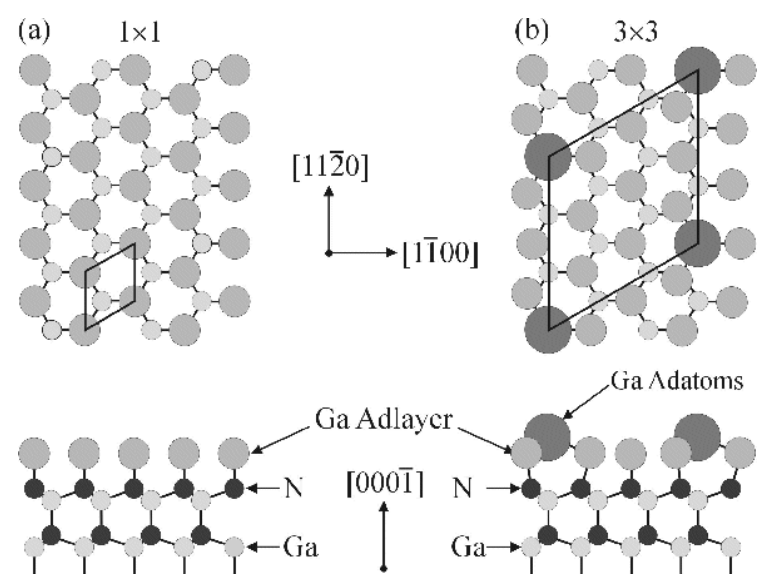

Figure 4. Schematic view of structures determined for the (a) $1 \times 1$ Ga adlayer and (b) $3 \times 3$ adatom-on-adlayer reconstructions of $\mathrm{GaN}(000 \overline{1})$. For the $3 \times 3$ structure, the lateral (in-plane) displacement of the adlayer atoms bonded to the Ga adatom is $0.51 \AA$ away from the adatom. All other lateral or vertical displacements of the adlayer atoms are less than $0.1 \AA$. (From [30]).

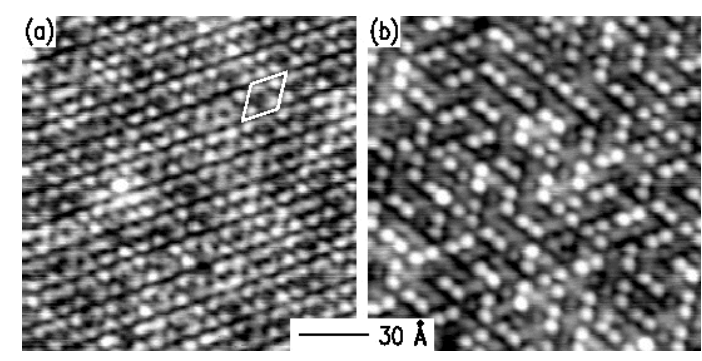

Figure 5. Simultaneously-acquired dual bias images of the $5 \times 5$ reconstruction. Sample biases are $+1.0 \mathrm{~V}$ and $-1.0 \mathrm{~V}$ with gray scale ranges of $0.5 \AA$ and $0.9 \AA$ for (a) and (b), respectively. (From [36]).

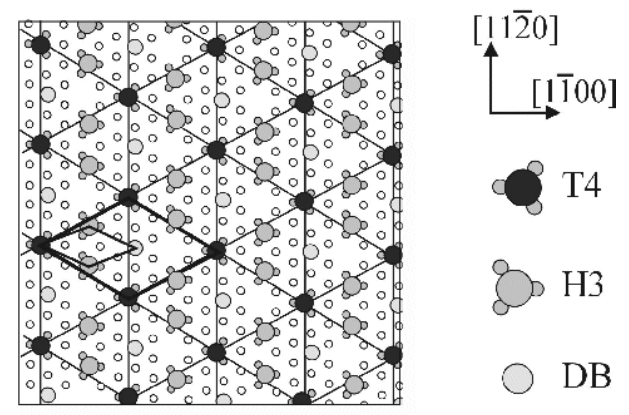

Figure 6. Structural model for the $5 \times 5$ reconstruction. Gaadatoms in $\mathrm{T} 4$ sites and $\mathrm{N}$-adatoms in $\mathrm{H} 3$ sites are shown by large black and large grey circles respectively. The small open circles in the diagram represent the $\mathrm{Ga}$ rest atoms in the 2nd layer. In the locations where the small open circles are missing, $\mathrm{Ga}$ vacancies occur. The $\mathrm{N}$ atoms in the 3rd layer are not shown. The light grey circle labelled DB (dangling bond) is a particular Ga rest atom site. In an alternative model, this site could conceivably contain another adatom in a nearby $\mathrm{T} 4$ or $\mathrm{H} 3$ site. (From [36]).

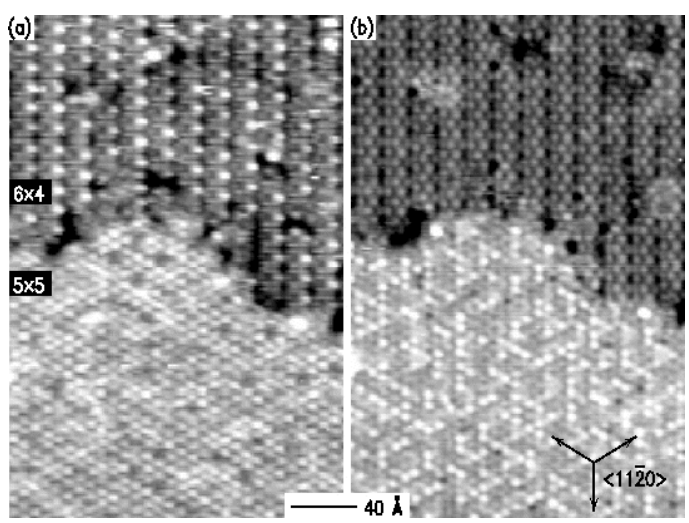

Figure 7. Dual bias images of the $5 \times 5$ and $6 \times 4$ reconstructions. The average height difference between the two reconstructions is $0.3 \AA$ for empty states (+1.0 V sample voltage) shown in (a) and $0.4 \AA$ for filled states ( $-1.0 \mathrm{~V}$ sample voltage) shown in (b), with the $5 \times 5$ being higher in each case. In both images the total gray scale range is about $1.3 \AA$. (From [36]). 

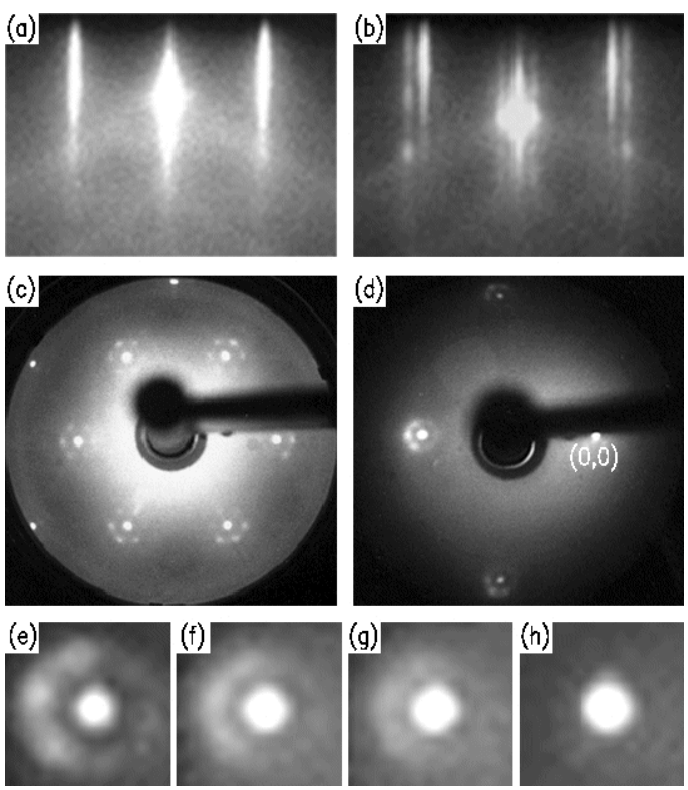

Figure 8. $1 \times 1$ RHEED pattern for $\mathrm{GaN}(0001)$ during growth, (a), and after cooling to below $350^{\circ} \mathrm{C}$, (b), where it converts to a $1+1 / 6$ pattern. The $1+1 / 6$ LEED pattern $\left(E_{i n c}=100 \mathrm{eV}\right)$ is shown in (c). For most " $1 \times 1$ " surfaces (see text), a $1+1 / 12$ pattern is observed below $200^{\circ} \mathrm{C}$, as shown in (d) $\left(E_{\text {inc }}=40 \mathrm{eV}\right)$. LEED in vicinity of $(0,1)$ spot $\left(E_{i n c}=40 \mathrm{eV}\right)$ at various temperatures: (e) $\mathrm{RT}-100^{\circ} \mathrm{C}$, (f) $100-150^{\circ} \mathrm{C}$, (g) $150-200^{\circ} \mathrm{C}$, and (h) above $350^{\circ} \mathrm{C}$. (From [33]).

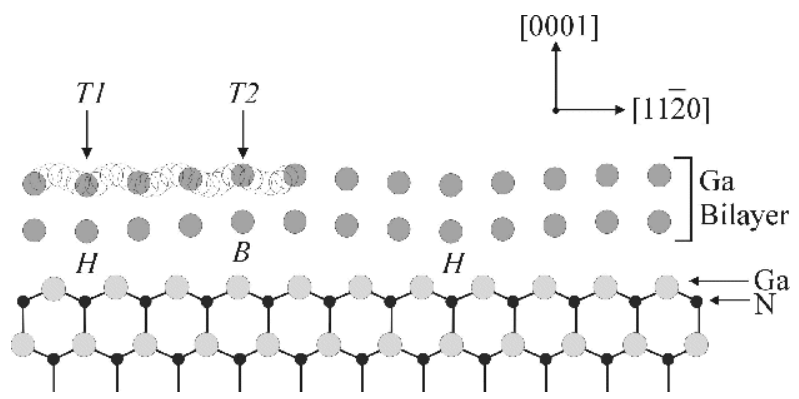

Figure 9. Side view of a possible structural model for the " $1 \times 1$ " surface (at a given instant in time) consisting of $2.7 \mathrm{ML}$ of Ga sitting on top of the Ga-terminated bilayer. The empty circles represent the various possible positions of first-layer $\mathrm{Ga}$ atoms plotted with respect to each of several $\mathrm{GaN}$ unit cells, illustrate the time-averaged height of the first layer $\mathrm{Ga}$ atoms and thus the $1 \times 1$ contour which the STM tip will follow. At a given instant in time, however, this incommensurate structure will manifest itself in diffraction as satellites surrounding the integral order peaks. (From [33]). (a) Laterally contracted bilayer model (time average)

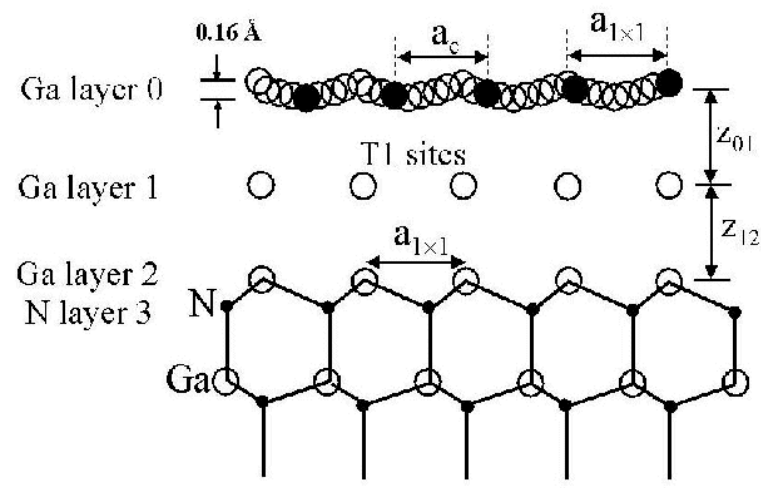

Figure 10a. A schematic representation of a laterally contracted Ga bilayer above a Ga-terminated (0001) substrate. The average separations between layers are $\mathrm{z}_{12}=2.54 \AA$ and $\mathrm{z}_{01}=$ $2.37 \AA$. The filled and open circles in layer 0 represent a time averaged image of the Ga atoms. The filled circles in layer 0 correspond to the positions at a particular time. The time averaged vertical corrugation of layer 0 is approximately 0.16 $\AA$. Note: In this projection the laterally contracted monolayer (layer 0 ) has been rotated by $30^{\circ}$ for ease of viewing. (From [46]).

\section{(b) registry $\mathrm{B}$}

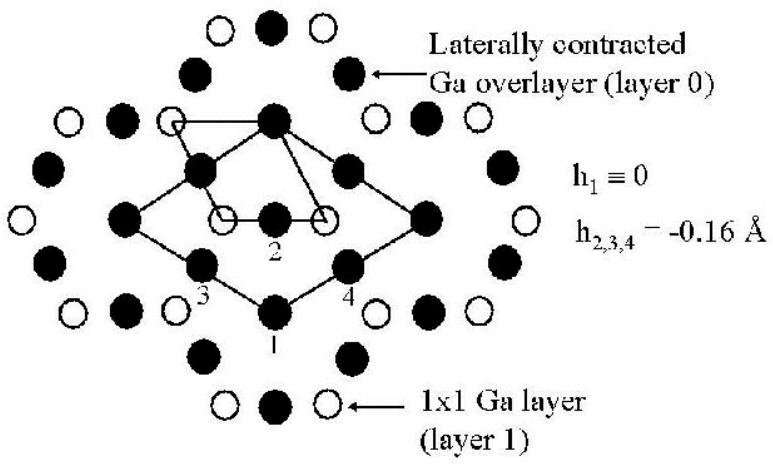

Figure 10b. Projection on the c-plane of the top two layers for the registry $B$ structure discussed in the text. Open circles represent a $1 \times 1$ adlayer of atoms located in T1 sites. (From [46]). 


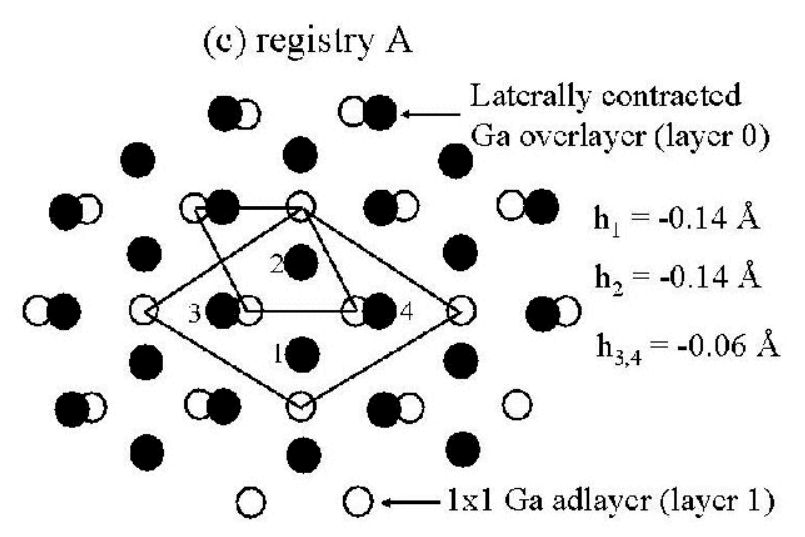

Figure 10c. Projection on the c-plane of the top two layers for registry $A$. The heights (h) of the Ga atoms in layer 0 , relative to that of atom 1 in registry $B$ are listed. (From [46]).

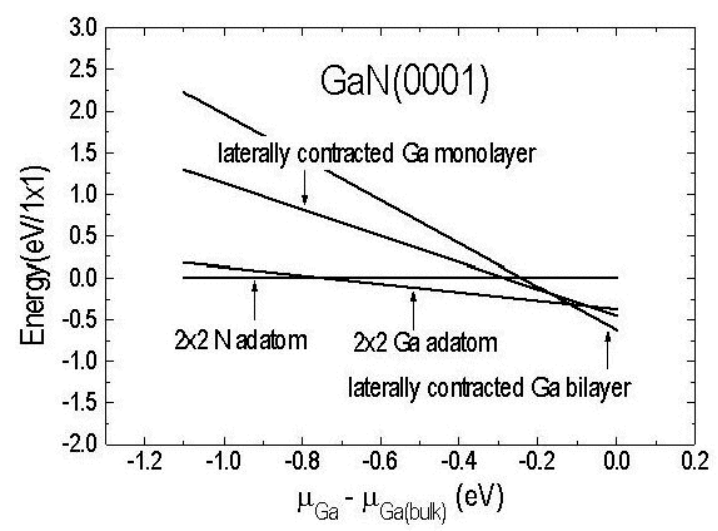

Figure 11. Relative energies of the surfaces are plotted as a function of the $\mathrm{Ga}$ chemical potential. For Ga-rich conditions the most stable structure is the laterally contracted Ga bilayer. (From [46]).
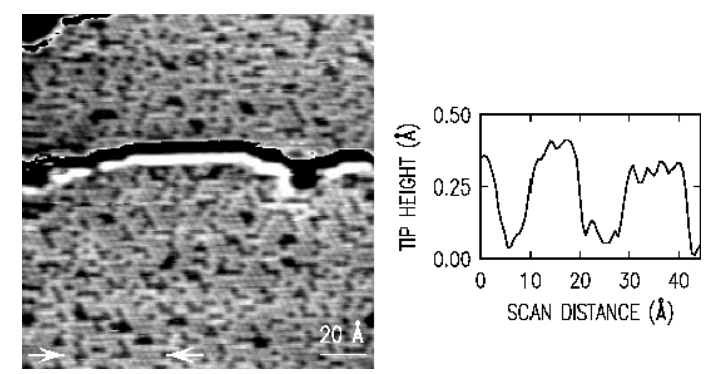

Figure 12. STM image of the $\operatorname{InGaN}(000 \overline{1})$ surface, obtained from a sample with negligible indium incorporation in the bulk. The image was acquired with sample bias voltage of $-0.5 \mathrm{~V}$ and tunnel current of $0.075 \mathrm{nA}$. The grey scale range is $0.6 \AA$ for each terrace. A line cut, taken at the position of the arrows in the figure, is shown on the right side of the figure. (From [50]).

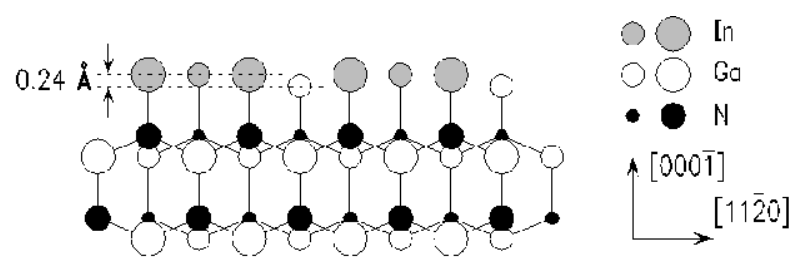

Figure 13. Schematic view of the InGaN surface in an (1100) projection, showing theoretical results for atomic positions. The surface adlayer consists of $75 \%$ In plus $25 \% \mathrm{Ga}$, in a $2 \times 2$ arrangement. (From [50]). 


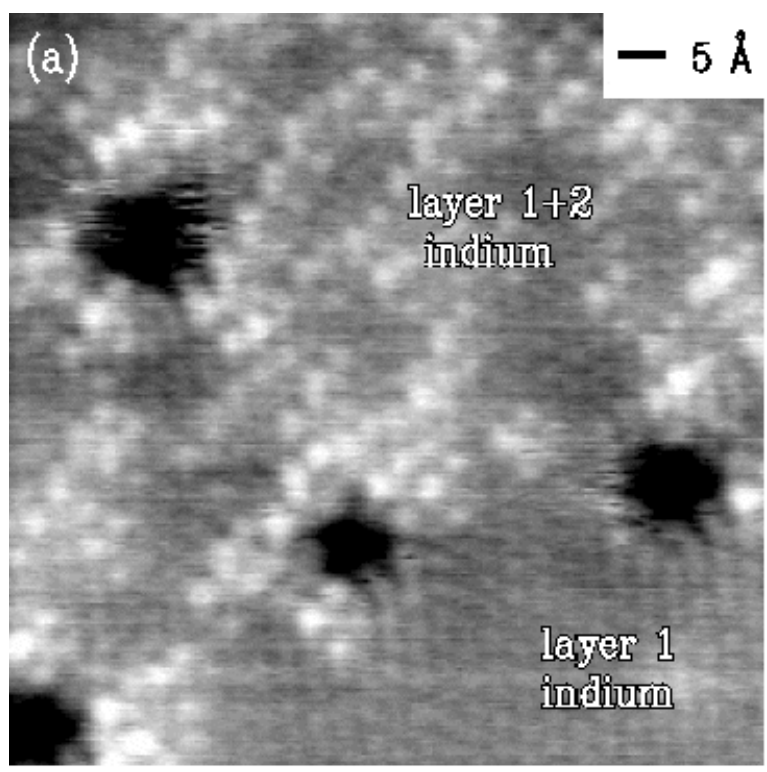

00000-layer 1-00000-In

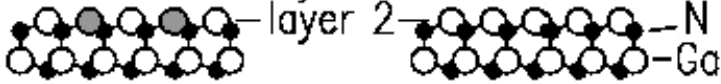

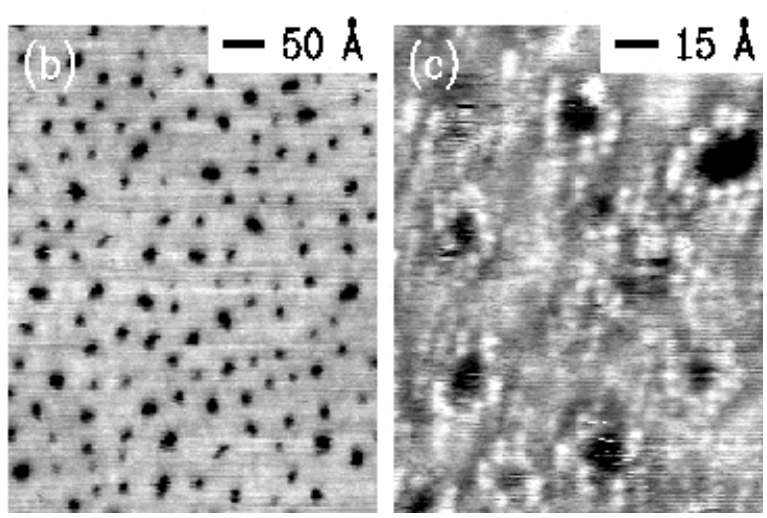

Figure 14. STM images of $\operatorname{InGaN}(0001)$ surface: (a) surface containing $0.9 \pm 0.2 \mathrm{ML}$ of indium. Image was acquired at a sample voltage of $+1.0 \mathrm{~V}$. Grey scale range is $0.6 \AA$. Different regions of the sample are labeled according to their In occupation, layer 1 or layer $1+2$, as pictured below on the image on the right- and left-hand sides respectively. (b) and (c) Surface containing 1.4 $\pm 0.2 \mathrm{ML}$ of indium. Images were acquired at sample voltages of (a) $-2.0 \mathrm{~V}$ and (b) $+1.5 \mathrm{~V}$. The entire surface consists of the vacancy island structure. Grey scale range is $0.6 \AA$ for (b) and (c). Tunnel current is $0.075 \mathrm{nA}$ for all images. (From [51]).

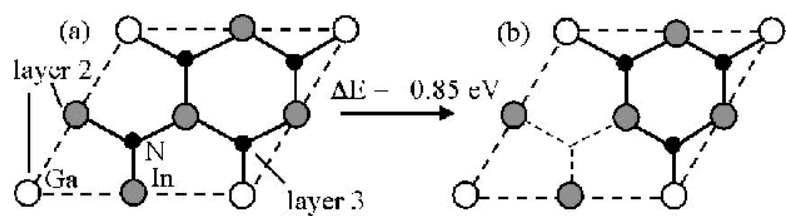

$7 / 4$ structure

$7 / 4+\mathrm{N}$-vaceancy structure

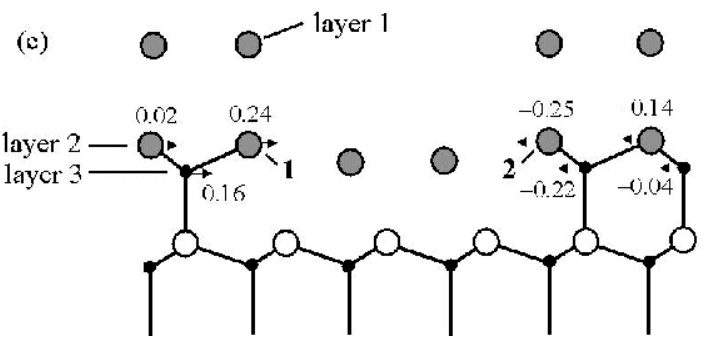

Figure 15. (a) Top view of the $7 / 4$ structure comprised of $7 / 4$ ML of In. In this $2 \times 2$ structure there is $3 / 4 \mathrm{ML}$ of In and 1/4 ML Ga in layer 2. Layer 1 (not shown) contains $1 \mathrm{ML}$ of In atoms. (b) Top view of the $7 / 4+\mathrm{N}$-vacancy structure, obtained from the $7 / 4$ structure by removing $N$ atoms from layer 3 . (c) Side view of a trench created by removing three rows of $\mathrm{N}$ atoms (layer 3) and two rows of In atoms (layer 1). Formation of this trench leads to substantial lateral displacements of the atoms in layers 2 and 3, shown in $\AA$. Indium atoms in sites 1 and 2 are bonded to 1 and $2 \mathrm{~N}$-atoms respectively. (From [51]).

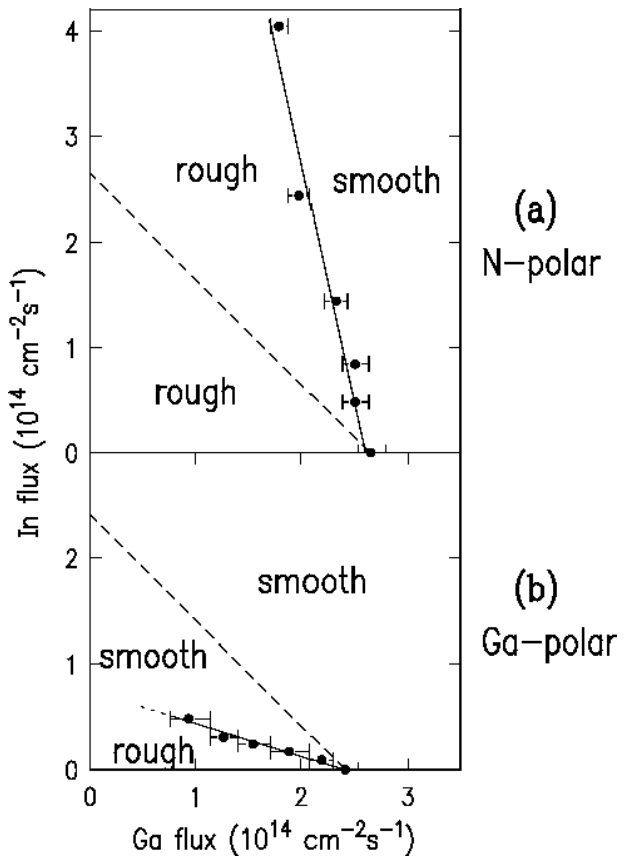

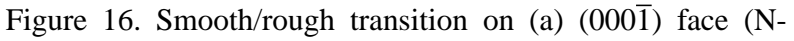
polar) and (b) (0001) face (Ga-polar). Nitrogen flux was fixed in both experiments. Substrate temperature was $600^{\circ} \mathrm{C}$. Experimental data is shown with dots, each with an error bar. A dashed line is shown in each figure for comparison denoting the line with constant total metal flux. To the right of the transition lines (solid lines) the growth is smooth. (From [52]). 

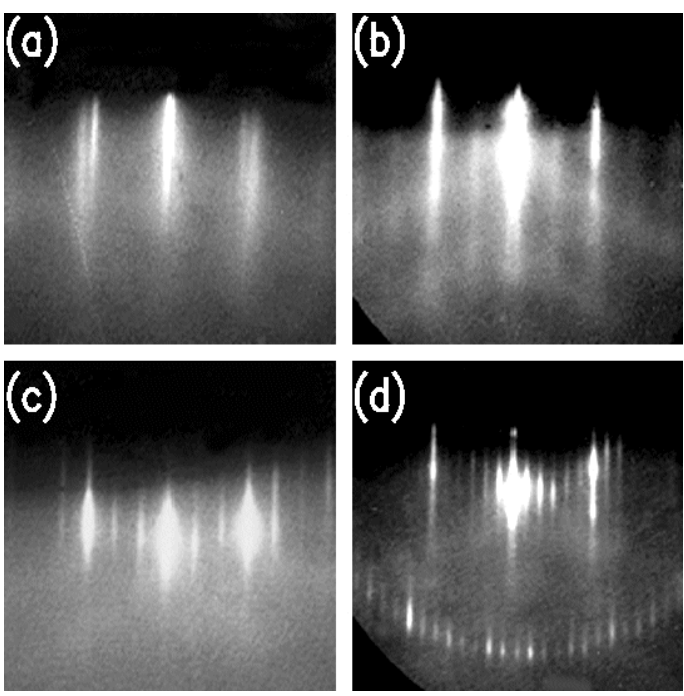

Figure 17. RHEED patterns seen after cooling of GaN films. Non-inverted surface: (a) " $1 \times 1$ " and (b) $2 \times 2$. After $\mathrm{Mg}$ exposure, inverted surface: (c) $3 \times 3$ and (d) $6 \times 6$. (From [60]). 

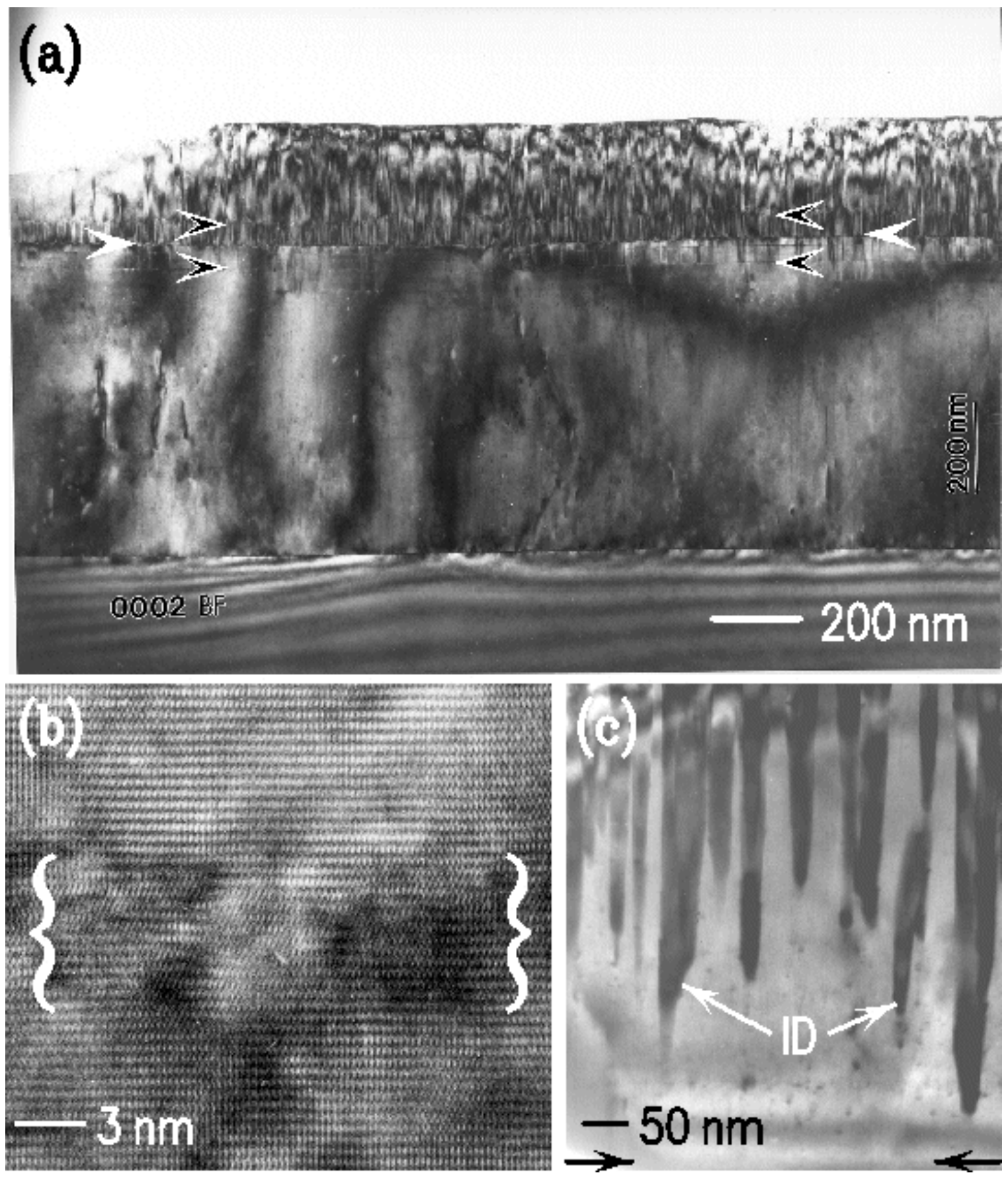

Figure 18. (a) Bright field TEM image in (0002) two-beam condition of a MBE-grown GaN film grown on SiC showing inversion boundary labeled with solid arrowheads and growth interrupts labeled with hollow arrowheads. (b) High-resolution image of the same sample as in (a). Brackets indicate the region of the inversion boundary. (c) Bright field (0002) two-beam TEM image of a MOCVD-grown GaN film showing inversion domains (ID). Regrowth interface is indicated by arrows. (From [60]). 


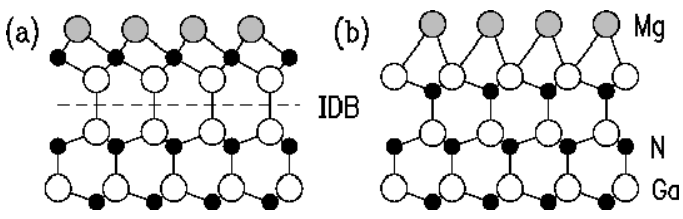

Figure 19. (a) Structural model of a c-plane inversion domain boundary (IDB) induced by $\mathrm{Mg}$ atoms. (b) Noninverted structure. Both models are shown in a $(11 \overline{2} 0)$ projection. Height of the $\mathrm{Mg}$ layer above the underlying atomic plane is $1.29 \AA$ and $2.43 \AA$ for inverted and noninverted structures respectively. (From [60]).
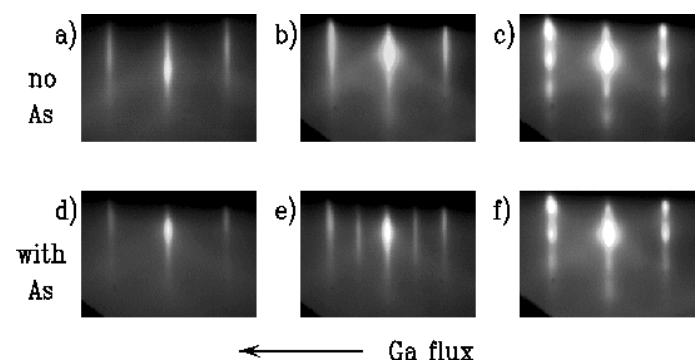

Figure 20. (a)-(c) RHEED patterns of Ga-polar films during growth without any arsenic, as a function of decreasing Ga flux. (d)-(f) A similar progression of RHEED patterns in the presence of arsenic with a BEP of $1 \times 10^{-9}$ Torr. (From [19]). 

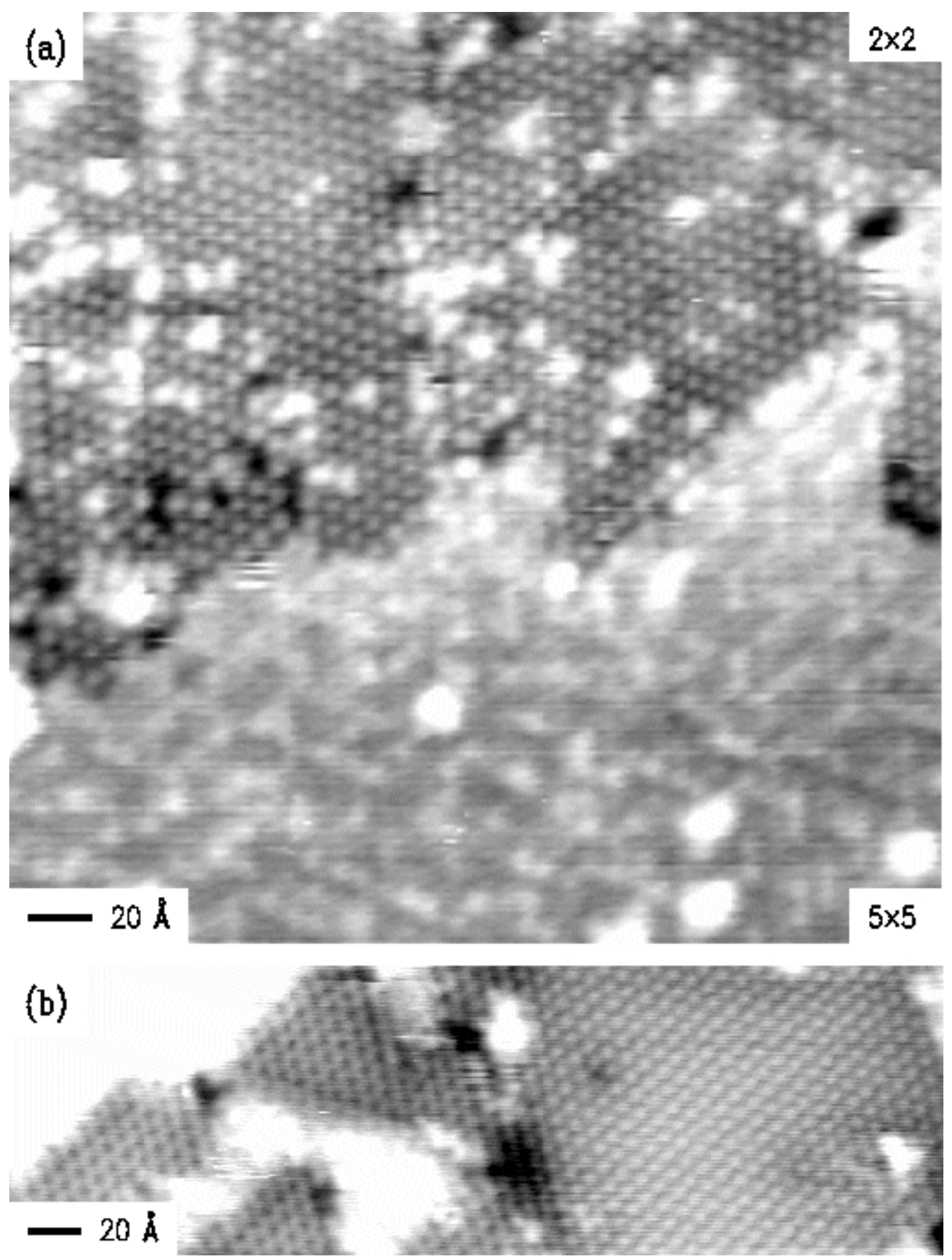

Figure 21. STM images of GaN(0001) surface exposed to $\approx 0.5 \mathrm{ML}$ of silicon. (a) Surface region showing Si-induced $2 \times 2$ reconstruction and the $5 \times 5$ reconstruction of the bare surface. (b) Two different types of domains (seen on the left and right sides of the image) of the $2 \times 2$ structures. Images were acquired with sample bias voltages of $-2.5 \mathrm{~V}$ and $-2.0 \mathrm{~V}$, respectively, and are shown with gray-scale ranges of 1.3 and $1.0 \AA$, respectively. (From [65]). 

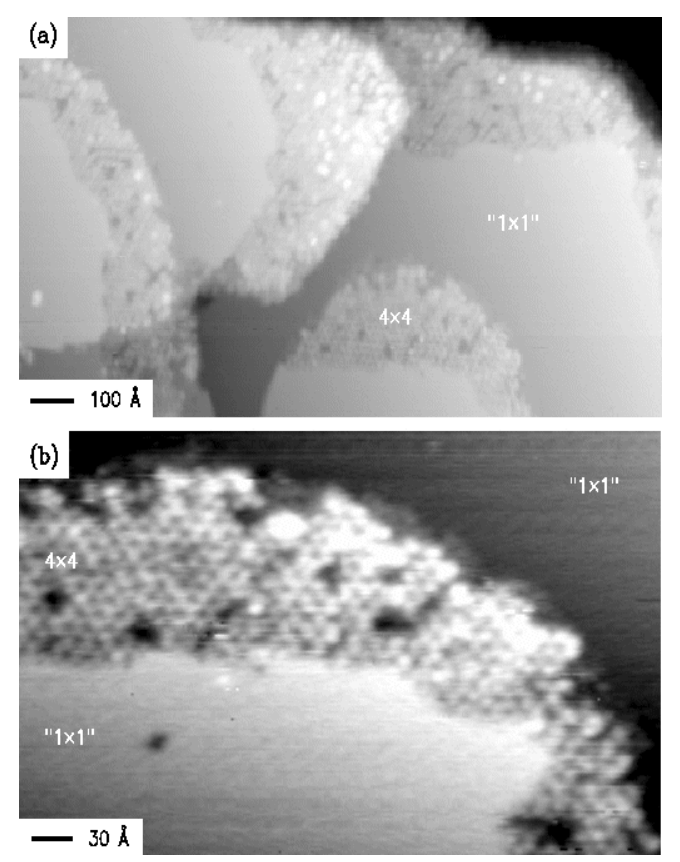

Figure 22. STM image of a $\mathrm{GaN}(0001)$ surface following $\approx 1$ ML silicon exposure. (a) Large scale image displaying terraces of " $1 \times 1$ " reconstruction with $4 \times 4$ structure seen at the terrace edges. (b) High resolution view of $4 \times 4$ structure near a terrace edge. Images were both acquired with a sample bias voltages of $+2.0 \mathrm{~V}$, and are shown with gray-scale ranges of 13 and $2.1 \AA$, respectively. (From [65]).

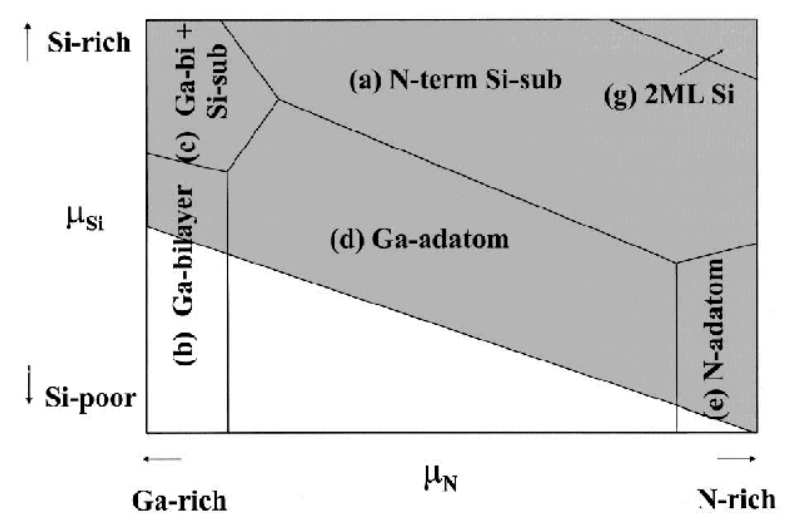

Figure 23. Phase diagram showing the energetically stable structures determined from first-principles calculations as a function of both $\mathrm{Si}\left(\mu_{\mathrm{Si}}\right)$ and $\mathrm{N}\left(\mu_{\mathrm{N}}\right)$ chemical potentials. The atomic geometry of these structures is shown in Figure 24. The limit at $\mathrm{N}$-rich conditions is $\mu_{\mathrm{Si}}=1 / 3 \Delta \mathrm{H}_{\mathrm{f}}\left(\mathrm{Si}_{3} \mathrm{~N}_{4}\right)$ and at Garich conditions is $\mu_{\mathrm{Si}}=1 / 3 \Delta \mathrm{H}_{\mathrm{f}}\left(\mathrm{Si}_{3} \mathrm{~N}_{4}\right)-4 / 3 \Delta \mathrm{H}_{\mathrm{f}}(\mathrm{GaN})$. $\Delta \mathrm{H}_{\mathrm{f}}\left(\mathrm{Si}_{3} \mathrm{~N}_{4}\right)=-3.32 \mathrm{eV}$ and $\Delta \mathrm{H}_{\mathrm{f}}(\mathrm{GaN})=-1.24 \mathrm{eV}$ are the calculated formation enthalpies of $\mathrm{Si}_{3} \mathrm{~N}_{4}$ and $\mathrm{GaN}$ bulk, respectively. The shaded area shows the region where all structures are unstable against the formation of $\mathrm{Si}_{3} \mathrm{~N}_{4}$. (From [66]).

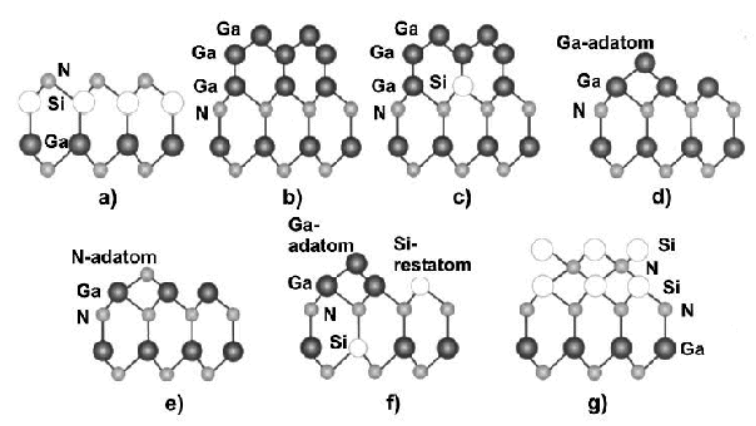

Figure 24. Schematic sideview of the energetically favorable structures for bare and Si-covered $\mathrm{GaN}(0001)$ surfaces. (a) $\mathrm{N}$ terminated with a Si subsurface, (b) Ga bilayer, (c) Ga bilayer with a $\mathrm{Si}$ subsurface, (d) Ga adatom, (e) N adatom, (f) $\mathrm{Ga}$ adatom with a Si subsurface, and (g) 2 ML of Si. See, also, Figure 23. (From [66]).

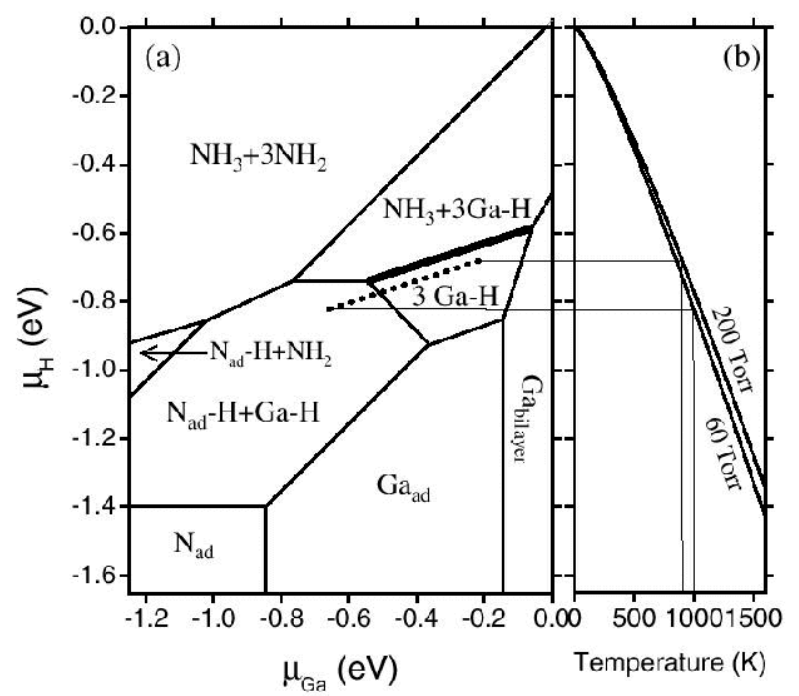

Figure 25. (a) Phase diagram for the $\mathrm{GaN}(0001)$ surface in the presence of $\mathrm{H}$, as a function of $\mu_{\mathrm{Ga}}$ and $\mu_{\mathrm{H}} \cdot \mu_{\mathrm{H}}=0$ corresponds to $\mathrm{H}$ molecules at $\mathrm{T}=0 ; \mu_{\mathrm{Ga}}=0$ corresponds to bulk Ga. Dots indicate experimental data from Ref. [14]; within the error bars, these data agree with the calculated $\mathrm{NH}_{3}+3 \mathrm{Ga}-\mathrm{H} / 3 \mathrm{Ga}-$ $\mathrm{H}$ phase boundary highlighted by the thicker line. Note that the $V_{\mathrm{Ga}}+6 \mathrm{H}$ structure is stoichiometrically and energetically equivalent to $\mathrm{NH}_{3}+3 \mathrm{Ga}-\mathrm{H}$. (b) temperature dependence of $\mu_{\mathrm{H}}$ for two different pressures. (From [80]). 


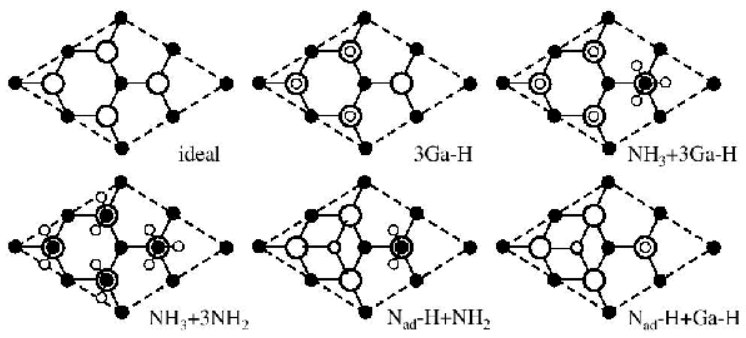

Figure 26 . Schematic top view of prevalent $2 \times 2$ reconstructions for $\mathrm{GaN}(0001)$ surfaces. Large open circles represent $\mathrm{Ga}$ atoms, solid circles N, and small open circles H. (From [80]).

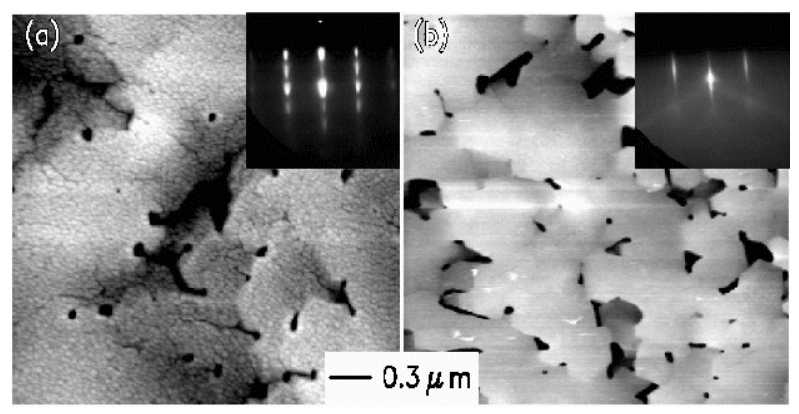

Figure 27. AFM images of GaN films grown under (a) Ga-rich conditions, and (b) N-rich conditions. The insets shows the corresponding RHEED patterns with the electron beam along (1120). (From [61]). 